TRANSACTIONS OF THE

AMERICAN MATHEMATICAL SOCIETY

Volume 360, Number 9, September 2008, Pages 4777-4814

S 0002-9947(08)04464-4

Article electronically published on April 8, 2008

\title{
STABILITY OF STATISTICAL PROPERTIES IN TWO-DIMENSIONAL PIECEWISE HYPERBOLIC MAPS
}

\author{
MARK F. DEMERS AND CARLANGELO LIVERANI
}

\begin{abstract}
We investigate the statistical properties of a piecewise smooth dynamical system by directly studying the action of the transfer operator on appropriate spaces of distributions. We accomplish such a program in the case of two-dimensional maps with uniformly bounded second derivative. For the class of systems at hand, we obtain a complete description of the SRB measures, their statistical properties and their stability with respect to many types of perturbations, including deterministic and random perturbations and holes.
\end{abstract}

\section{INTRODUCTION}

In recent years, many works have sought to establish in the hyperbolic setting the functional analytic approach developed for one-dimensional piecewise expanding maps.

This strategy completely avoids any attempt to code the system and directly studies the transfer operator on an appropriate Banach space (in the expanding case, the functions of bounded variation). Roughly speaking, the approach is to first obtain a priori control on the smoothing properties of the transfer operator $[\mathrm{LY}$, then infer from those that the transfer operator is quasi-compact and that its peripheral spectrum provides abundant information about the statistical properties of the system $[\mathrm{K}]$, and finally show that such a picture is stable for a large class of perturbations $\mathrm{BY}, \mathrm{KL}$. See [B1] for a detailed explanation of the above ideas and complete references and L2 for an apology.

Such a point of view was successfully extended to multi-dimensional expanding maps $[\mathrm{S}, \mathrm{Bu}, \mathrm{T1}, \mathrm{T2}, \mathrm{BK}$, but its application to the hyperbolic setting has been lacking until recently. Notwithstanding some partial successes [Ba, L1, R1, R2, R3,

Received by the editors July 17, 2006 .

2000 Mathematics Subject Classification. Primary 37D50, 37D20, 37C30.

Key words and phrases. Hyperbolic, piecewise discontinuous, transfer operator, decay of correlations, open systems.

The authors would like to thank the Institut Henri Poincaré where part of this work was done (during the trimester Time at Work). Also the authors enjoyed partial support from M.I.U.R. (Cofin 05-06 PRIN 2004028108). The first author was partially supported by NSF VIGRE Grant DMS-0135290 and by the School of Mathematics of the Georgia Institute of Technology. Finally, the second author would like to warmly thank G. Keller with whom, several years ago, he had uncountably many discussions on these types of problems. Although we were not able to solve the problem at the time, as the technology was not ripe yet, the groundwork we did has been precious for the present work. 
the first paper in which the above approach was systematically implemented in all its aspects was BKL, in which the authors studied Anosov diffeomorphisms. Such results have subsequently been dramatically improved in a series of papers GL, B2, BT, L3] of which certainly we have not seen the end.

In spite of the fact that in one dimension the approach was developed to overcome the problem of discontinuities, the case of piecewise hyperbolic systems has eluded attempts to treat it along such lines (with the partial exception of [L1]). Consequently, as far as hyperbolic systems with discontinuities are concerned, the only available approaches are $[\mathrm{P}$ and $\mathrm{Y}$ (and the generalizations by Chernov $\mathrm{Ch}$. and Chernov, Dolgopyat $\mathrm{CD}$ of Young's approach in the case of billiards; see $\mathrm{CY}$ ] for a review). Such approaches require a very deep preliminary understanding of the regularity properties of the invariant foliations and are not well-suited to the study of perturbations of the systems under consideration.

The present paper makes a first step in overcoming the difficulty of discontinuities by showing that in the two-dimensional case the functional analytic approach can be carried out successfully. We are confident that this approach can be extended to billiards and higher-dimensional systems.

The outline of the paper is as follows. In Section 3, we describe functional spaces on which we establish the quasi-compactness of the transfer operator in Section 4. This is the key result of the paper from which all the rest follow.

In Section 5. we show that there exists a precise relation between the spectral picture of the transfer operator and the statistical properties of the system. More precisely, the peripheral spectrum corresponds to the ergodic decomposition with respect to the physical measures, so a complete description of the SRB measures for the system is obtained.

The rest of the spectrum is connected to the finer statistical properties such as the decay of correlations, which is proven to be exponential for mixing systems, the Central Limit Theorem, the power spectrum and the Ruelle resonances. Although the decay of correlations and CLT are already known for systems with a slightly more restrictive class of singularities (see $[\mathrm{Y}]$ ), the current approach presents a unified framework for these results and adds to them a detailed understanding of the power spectrum and Ruelle resonances not previously available. (See Ru1, Ru2, PP1, PP2 for a discussion of Ruelle resonances in Axiom A systems.)

In addition, we answer questions concerning the stability of these statistical properties with respect to both deterministic and random perturbations, as well as those obtained by introducing small holes into the system. We prove that the stability is of a very strong nature: all the statistical properties, from the invariant measures to the rate of decay of correlations to the Ruelle resonances, vary continuously with the perturbation. The proofs of these results are contained in Section 6 ,

Contrary to GL, the spaces introduced here do not allow an extensive study of the influence of the smoothness of the system on its statistical properties. This may depend on the class of systems under investigation: contrary to the smooth case in which the degree of smoothness determines the size of the essential spectrum, it is conceivable that there is no difference between piecewise $\mathcal{C}^{2}$ and piecewise $\mathcal{C}^{r}$ systems. On the other hand there may be a difference that is not captured by our spaces.

Finally, note that the paper tackles the problem, left open in GL, of how to define spaces with Hölder regularity in the unstable direction. 
Remark 1.1. A remarkable aspect of the present approach is that it bypasses completely the detailed, and extremely laborious, study of the smoothness properties of the invariant foliations, their holonomies and the local ergodicity theorems (albeit restricted to the uniformly hyperbolic case). Accordingly, it provides an extremely direct way to obtain very strong results, as testified by the length of the present, essentially self-contained, paper.

Convention 1.2. In this paper we will use $C$ to denote a generic constant depending only on the dynamical systems $(\mathcal{M}, T)$, while $C_{a, b, c \ldots}$ will depend only on $(\mathcal{M}, T)$ and the parameters $a, b, c, \ldots$. Accordingly, the actual value of such constants may vary from one occurrence to the next.

\section{Setting, DEFinitions AND RESUlts}

Let $\mathcal{M}$ be a compact two-dimensional Riemannian manifold, possibly with boundary and not necessarily connected, and let $T: \mathcal{M} \circlearrowleft$ be a piecewise uniformly hyperbolic map in the following sense. We assume that there exist a finite number of pairwise disjoint open regions $\left\{\mathcal{M}_{i}^{+}\right\}$such that $\bigcup_{i} \overline{\mathcal{M}_{i}^{+}}=\mathcal{M}$ and the boundaries of $\mathcal{M}_{i}^{+}$are piecewise $\mathcal{C}^{1}$ curves of finite length. We define $\mathcal{M}_{i}^{-}=T\left(\mathcal{M}_{i}^{+}\right)$and require that $\bigcup_{i} \overline{\mathcal{M}_{i}^{-}}=\mathcal{M}$. We refer to the sets $\mathcal{S}^{ \pm}:=\mathcal{M} \backslash \bigcup_{i} \mathcal{M}_{i}^{ \pm}$as the singularity sets for $T$ and $T^{-1}$, respectively. We assume that $T \in \operatorname{Diff}^{2}\left(\mathcal{M} \backslash \mathcal{S}^{+}, \mathcal{M} \backslash \mathcal{S}^{-}\right)$and that on each $\mathcal{M}_{i}^{+}, T$ has a $\mathcal{C}^{2}$ extension to $\overline{\mathcal{M}_{i}^{+}}$.

On each $\mathcal{M}_{i}, T$ is uniformly hyperbolic: i.e., it admits two continuous $D T$ strictly-invariant families of cones $C^{s}$ and $C^{u}$ defined on all of $\mathcal{M} \backslash\left(\mathcal{S}^{+} \cup \partial \mathcal{M}\right)$ which satisfy 1

$$
\begin{aligned}
& \lambda:=\inf _{x \in \mathcal{M} \backslash \mathcal{S}^{+}} \inf _{v \in C^{u}} \frac{\|D T v\|}{\|v\|}>1, \\
& \mu:=\inf _{x \in \mathcal{M} \backslash \mathcal{S}^{+}} \inf _{v \in C^{s}} \frac{\|D T v\|}{\|v\|}<1, \\
& \mu_{+}^{-1}:=\inf _{x \in \mathcal{M} \backslash \mathcal{S}^{-}} \inf _{v \in C^{s}} \frac{\left\|D T^{-1} v\right\|}{\|v\|}>1 .
\end{aligned}
$$

In Section 3.1, we define narrow cones with the same names and refer to them as the stable and unstable cones of $T$, respectively. We assume that the tangent vectors to the singularity curves in $\mathcal{S}^{-}$are bounded away from $C^{s}$. Note that this class of maps is similar to that studied in $[\mathrm{Y}, \mathrm{P}]$; see also [LW] for the symplectic case.

Remark 2.1. We can replace the condition that the singularity curves be transverse to $C^{s}$ by the more general assumption (H1) of Section 2.5 (replacing $\partial H$ with $\mathcal{S}^{-}$), thus allowing singularities which are in places tangent to the stable direction. The estimates of Section 6.3 imply that Proposition 2.7 and Theorem 2.8 hold with this weaker condition on the singularities of $T$ as long as we choose $\beta \leq \alpha / 2$ in the definition of the strong unstable norm (2.4). We do not do this, however, since this restriction on $\beta$ makes less optimal our estimates on the essential spectral radius (see Remark [5.9).

\footnotetext{
${ }^{1}$ Note that the strict invariance of the cone field together with the smoothness properties of the map implies that the stable and unstable directions are well-defined for each point whose trajectory does not meet a singularity line.
} 
Remark 2.2. Although the class of maps $T$ which we consider does not contain billiards, it does contain piecewise toral automorphisms and a broad class of piecewise hyperbolic nonlinear maps with bounded derivative.

Denote by $\mathcal{S}_{n}^{-}$the set of singularity curves for $T^{-n}$ and by $\mathcal{S}_{n}^{+}$the set of singularity curves for $T^{n}$. Let $M(n)$ denote the maximum number of singularity curves in $\mathcal{S}_{n}^{-}$which intersect at a single point. We make the following assumption regarding the singularities of $T$ :

$$
\begin{aligned}
& \text { There exist } \alpha_{0}>0 \text { and an integer } n_{0}>0 \text {, such that } \lambda \mu^{\alpha_{0}}>1 \text { and } \\
& \left(\lambda \mu^{\alpha_{0}}\right)^{n_{0}}>M\left(n_{0}\right) .
\end{aligned}
$$

Condition (P1) can always be satisfied if $M(n)$ has polynomial growth (as is the case with billiards); however, since (P1) is required only for some fixed $n_{0}$, it is not necessary to control $M(n)$ for all $n$ in order to verify the condition.

Remark 2.3. If property (P1) holds for $\alpha_{0}$, then it holds for all $0<\alpha<\alpha_{0}$ with the same $n_{0}$. Notice also that $M\left(k n_{0}\right) \leq M\left(n_{0}\right)^{k}$, which implies that the inequality in (P1) can be iterated to make $\left(\lambda \mu^{\alpha_{0}}\right)^{-k n_{0}} M\left(k n_{0}\right)$ arbitrarily small once (P1) is satisfied for some $n_{0}$.

In Section 3.1 we will define a set of admissible leaves $\Sigma$, close to the stable direction, on which we will define our norms. For a leaf $W \in \Sigma$, let $L_{n}$ denote the number of smooth connected components of $T^{-n} W$. For a fixed $N$, by shrinking the maximum length $2 \delta$ of leaves in $\Sigma$, we can require that $L_{N} \leq M(N)+1$. This implies that choosing $N=k n_{0}$, we can make $\left(\lambda \mu^{\alpha_{0}}\right)^{-N} L_{N}$ arbitrarily small.

Convention 2.4. In what follows, we will assume that $n_{0}=1$. If this is not the case, we may always consider a higher iterate of $T$ for which this is so by assumption (P1). We refer to $L_{1}$ as $L$ and choose $\delta$ small enough that $L \lambda^{-1} \mu^{-\alpha_{0}}=: \rho<1$.

We write $D^{s}$ to denote differentiation in the stable direction and note that this direction is well-defined outside the set $\bigcup_{n \geq 0} \mathcal{S}_{n}^{+}$due to the uniform hyperbolicity of $T$.

For an admissible leaf $W \in \Sigma$, we will denote by $m$ the (unnormalized) Riemannian volume on $W$ and by $d(\cdot, \cdot)$ the distance along the leaf. We will often abbreviate $m(W)$ by $|W|$.

2.1. Transfer operator. The basic object of study in the present paper is the so-called transfer operator $\mathcal{L}$. Clearly, to make sense of an operator it is necessary to specify on which space it acts. In fact, the search for a good space is the main point of the present paper.

In the smooth case [GL, it is convenient to define the transfer operator acting on the space of distributions which turns out to contain all the relevant spaces. In this manner one can obtain all the relevant operators as restrictions of the original one.

In the present case it is not clear if there exists an appropriate ambient space $2^{2}$ We bypass this problem by defining the operator as acting between two scales of spaces.

For each $n \in \mathbb{N}$, let $\mathcal{K}_{n}$ be the set of connected components of $\mathcal{M} \backslash \mathcal{S}_{n}^{+}$. Recall that $\mathcal{C}^{1}(\bar{K}, \mathbb{R})$ is the set of functions $\varphi \in \mathcal{C}^{1}(\stackrel{\circ}{K}, \mathbb{R})$ which have a $\mathcal{C}^{1}$ extension in a

\footnotetext{
${ }^{2}$ Clearly the space of distributions will not do since if $\varphi$ is smooth, $\varphi \circ T$ may not be.
} 
neighborhood of $\bar{K}$. Let $\mathcal{C}_{\mathcal{S}_{n}^{+}}^{1}:=\left\{\varphi \in L^{\infty}(\mathcal{M}): \varphi \in \mathcal{C}^{1}(\bar{K}, \mathbb{R}) \forall K \in \mathcal{K}_{n}\right\}$ If $h \in\left(\mathcal{C}_{\mathcal{S}_{n}^{+}}^{1}\right)^{\prime}$ is an element of the dual of $\mathcal{C}_{\mathcal{S}_{n}^{+}}^{1}$, then $\mathcal{L}:\left(\mathcal{C}_{\mathcal{S}_{n}^{+}}^{1}\right)^{\prime} \rightarrow\left(\mathcal{C}_{\mathcal{S}_{n-1}^{+}}^{1}\right)^{\prime}$ acts on $h$ by

$$
\mathcal{L} h(\varphi)=h(\varphi \circ T) \quad \forall \varphi \in \mathcal{C}_{\mathcal{S}_{n-1}^{+}}^{1} .
$$

The above definition shows how the transfer operator acts on an abstract space of distributions, but often we will be concerned with its action on more concrete objects. Notice that since the sets $\mathcal{S}_{n}^{+}$are all of zero Lebesgue (Riemannian) measure, each signed measure absolutely continuous with respect to Lebesgue yields an element of $\left(\mathcal{C}_{\mathcal{S}_{n}^{+}}^{1}\right)^{\prime}$.

Remark 2.5. In what follows, we will identify a measure $h$ that is absolutely continuous with respect to Lebesgue with its density, which we will insist on calling $h$. Accordingly,

$$
h(\varphi)=\int_{\mathcal{M}} h \varphi d m
$$

where $m$ denotes Lebesgue measure on $\mathcal{M}$. Hence the space of measures absolutely continuous with respect to Lebesgue is canonically identified with $L^{1}(\mathcal{M}, \mathbb{R}, m)$.

With the above convention, $L^{1}(\mathcal{M}) \subset\left(\mathcal{C}_{\mathcal{S}_{n}^{+}}^{1}\right)^{\prime}$ for each $n \in \mathbb{N}$. One can then restrict $\mathcal{L}$ to $L^{1}$ and a simple computation shows that

$$
\mathcal{L}^{n} h=h \circ T^{-n}\left|D T^{n}\left(T^{-n}\right)\right|^{-1}
$$

for any $n \geq 0$ and any $h \in L^{1}(\mathcal{M}) 5$

2.2. Definition of the norms. We will define the required Banach spaces by closing $\mathcal{C}^{1}$ with respect to suitable norms.

The norms are defined via a set of admissible leaves $\Sigma$. Such leaves are essentially smooth curves roughly in the stable direction, their length is smaller than some $\delta$ and among them is defined a notion of distance $d_{\Sigma}$. Also, a notion of distance $d_{q}$ is defined among functions supported on such leaves. They are defined precisely in Section 3.1 ,

For $W \in \Sigma$ and $0 \leq \alpha, q \leq 1$, denote by $\mathcal{C}^{\alpha}(W, \mathbb{C})$ the set of continuous complexvalued functions on $W$ with Hölder exponent $\alpha$. Define the following norms:

$$
|\varphi|_{W, \alpha, q}:=|W|^{\alpha} \cdot|\varphi|_{\mathcal{C}^{q}(W, \mathbb{C})} .
$$

Given a function $h \in \mathcal{C}^{1}(\mathcal{M}, \mathbb{C})$, define the weak norm of $h$ by

$$
|h|_{w}:=\sup _{W \in \Sigma} \sup _{\substack{\varphi \in \mathcal{C}^{1}(W, \mathbb{C}) \\|\varphi|_{\mathcal{C}^{1}(W)} \leq 1}} \int_{W} h \varphi d m .
$$

\footnotetext{
${ }^{3}$ The space $\mathcal{C}_{\mathcal{S}_{n}^{+}}^{1}$ is a Banach space when equipped with the norm $\sup _{K \in \mathcal{K}_{n}}|\varphi|_{\mathcal{C}^{1}(K)}$.

${ }^{4}$ Given a square matrix $A$, by $|A|$ we mean $|\operatorname{det}(A)|$.

${ }^{5}$ Often the above is taken as the definition of the transfer operator, yet as will become clear in the following, $L^{1}$ is both too small and too large a space to be useful.
} 
Choose $\alpha, \beta, q<1$ such that $0<\beta \leq \alpha \leq 1-q \leq \alpha_{0} 6$ We define the strong stable norm as

$$
\|h\|_{s}:=\sup _{W \in \Sigma} \sup _{\substack{\varphi \in \mathcal{C}^{1}(W, \mathbb{C}) \\|\varphi|_{W, \alpha, q} \leq 1}} \int_{W} h \varphi d m
$$

and the strong unstable norm as

$$
\|h\|_{u}:=\sup _{\varepsilon \leq \varepsilon_{0}} \sup _{\substack{W_{1}, W_{2} \in \Sigma \\ d_{\Sigma}\left(W_{1}, W_{2}\right) \leq \varepsilon}} \sup _{\substack{\left|\varphi_{i}\right|_{\mathcal{C}^{1}\left(W_{i}, \mathbb{C}\right) \leq 1} \\ d_{q}\left(\varphi_{1}, \varphi_{2}\right) \leq \varepsilon}} \frac{1}{\varepsilon^{\beta}}\left|\int_{W_{1}} h \varphi_{1} d m-\int_{W_{2}} h \varphi_{2} d m\right|
$$

where $\varepsilon_{0}$ will be chosen later. We then define the strong norm of $h$ by

$$
\|h\|=\|h\|_{s}+b\|h\|_{u}
$$

where $b$ is a small constant chosen in Section 4

We define $\mathcal{B}$ to be the completion of $\mathcal{C}^{1}(\mathcal{M})$ in the strong norm and $\mathcal{B}_{w}$ to be the completion of $\mathcal{C}^{1}(\mathcal{M})$ in the weak norm.

Finally, let

$$
D_{n}:=\delta^{\alpha-1} \sup _{0 \leq k \leq n} \sup _{W \in \Sigma}|W|^{-\alpha} \int_{W}\left|D T^{-k}\right| d m
$$

and set $D_{*}=\limsup _{n \rightarrow \infty} D_{n}^{1 / n}$.

2.3. Statement of results. The first result gives a more concrete description of the above abstract spaces.

Lemma 2.6. For each $n \geq 0, \mathcal{B} \subset \mathcal{B}_{w} \subset\left(\mathcal{C}_{\mathcal{S}_{n}^{+}}^{1}\right)^{\prime}$.

Proof. This is an immediate consequence of Lemma 3.3 and the fact that $|\cdot|_{w} \leq$ $\|\cdot\|$.

In addition, the transfer operator is well-defined on the spaces $\mathcal{B}, \mathcal{B}_{w}$. In fact, the following more precise result is proven in Section 4 .

Proposition 2.7. There exists $\delta_{0}>0$ such that for all $h \in \mathcal{B}, \delta \leq \delta_{0}$ and $n \geq 0$,

$$
\begin{aligned}
\left|\mathcal{L}^{n} h\right|_{w} & \leq C D_{n}|h|_{w}, \\
\left\|\mathcal{L}^{n} h\right\|_{s} & \leq C \max \left\{\rho, \mu_{+}^{q}\right\}^{n} D_{n}\|h\|_{s}+C_{\delta} D_{n}|h|_{w}, \\
\left\|\mathcal{L}^{n} h\right\|_{u} & \leq C \lambda^{-\beta n} D_{n}\|h\|_{u}+C\left(D_{n}+L_{n} \lambda^{-n} \mu^{-\alpha n}\right)\|h\|_{s} .
\end{aligned}
$$

If we choose $1>\tau>\max \left\{\lambda^{-\beta}, \rho, \mu_{+}^{q}\right\}$, then there exists $N \geq 0$ such that

$$
\begin{aligned}
\left\|\mathcal{L}^{N} h\right\| & =\left\|\mathcal{L}^{N} h\right\|_{s}+b\left\|\mathcal{L}^{N} h\right\|_{u} \\
& \leq \frac{\tau^{N} D_{N}}{2}\|h\|_{s}+C_{\delta} D_{N}|h|_{w}+b \tau^{N} D_{N}\|h\|_{u}+b C\left(D_{N}+L_{N} \lambda^{-N} \mu^{-\alpha N}\right)\|h\|_{s} \\
& \leq \tau^{N} D_{N}\|h\|+C_{\delta} D_{N}|h|_{w}
\end{aligned}
$$

provided $b$ is chosen small enough with respect to $N$. The above represents the traditional Lasota-Yorke inequality once we show the $D_{n}$ are bounded. Probably

\footnotetext{
${ }^{6}$ Such inequalities are irrelevant for the definition of the spaces, but we introduce them here because they will be needed for the various Lasota-Yorke estimates of Section 4
} 
a direct argument could prove this fact, yet we find it easier to prove using a functional analytic argument.

The final ingredient in the strategy to prove the quasi-compactness of the operator $\mathcal{L}$ is the relative compactness of the unit ball of $\mathcal{B}$ in $\mathcal{B}_{w}$. This is proven in Lemma 3.5. It thus follows by standard arguments (B1]) that the essential spectral radius of $\mathcal{L}$ on $\mathcal{B}$ is bounded by $\tau D_{*}$, while the estimate for the spectral radius, contrary to the usual situation, is $D_{*}$ which, in general, could be larger than one. Nevertheless, a functional analytic argument (Lemma 5.3) shows that the spectral radius is one. As a consequence we know, a posteriori, that $D_{*}=1$, and this together with Lemma 5.1 implies that the $D_{n}$ are bounded (see Remark 5.4).

Our next results characterize the set of invariant measures in $\mathcal{B}$ and some of the statistical properties of $T$. Recall that an invariant probability measure $\mu$ is called a physical measure if there exists a positive Lebesgue measure invariant set $B_{\mu}$, with $\mu\left(B_{\mu}\right)=1$, such that, for each continuous function $f$,

$$
\lim _{n \rightarrow \infty} \frac{1}{n} \sum_{i=0}^{n-1} f\left(T^{i} x\right)=\mu(f) \quad \forall x \in B_{\mu} .
$$

Let $\Pi_{\theta}$ be the eigenprojector on $\mathbb{V}_{\theta}$, the eigenspace of $\mathcal{L}$ corresponding to eigenvalue $e^{2 \pi i \theta}$, and set $\mathbb{V}:=\bigoplus_{\theta} \mathbb{V}_{\theta}$. The following theorem is proved by the lemmas of Section 5 .

Theorem 2.8. The peripheral spectrum of $\mathcal{L}$ on $\mathcal{B}$ consists of finitely many cyclic groups. The maps $\left\{T^{n}\right\}_{n \in \mathbb{N}}$ admit only finitely many physical probability measures, they form a basis for $\mathbb{V}$ and the cycles correspond to the cyclic groups. In addition,

(1) If $\mu \in \mathbb{V}_{0}$ and $\mathcal{S}_{n, \epsilon}^{ \pm}$is an $\epsilon$-neighborhood of $\mathcal{S}_{n}^{ \pm}$, then $\mu\left(\mathcal{S}_{n, \epsilon}^{ \pm}\right) \leq C_{n} \epsilon^{\alpha}$ for all $n \in \mathbb{N}$. In particular, $\mu\left(\mathcal{S}_{n}^{ \pm}\right)=0$.

(2) Each element in $\mathbb{V}$ is a signed measure absolutely continuous with respect to the probability measure $\bar{\mu}:=\lim _{n \rightarrow \infty} \frac{1}{n} \sum_{i=0}^{n-1} \mathcal{L}^{i} 1$. In particular, all the physical measures are absolutely continuous with respect to $\bar{\mu}$.

(3) The supports of the physical measures correspond to the ergodic decomposition with respect to Lebesgue.

(4) For all $f \in \mathcal{C}^{0}(\mathcal{M}, \mathbb{R})$, the limit $f^{+}(x):=\lim _{n \rightarrow \infty} \frac{1}{n} \sum_{i=0}^{n-1} f \circ T^{i}(x)$ exists for $m$-almost-every $x$ and takes on only finitely many different values. If $\bar{\mu}$ is ergodic, then $f^{+}(x)=\int f d \bar{\mu}$ for $m$-almost-every $x$.

(5) If $(T, \bar{\mu})$ is ergodic, then 1 is a simple eigenvalue. If $\left(T^{n}, \bar{\mu}\right)$ is ergodic for all $n \in \mathbb{N}$, then one is the only eigenvalue of modulus one, $(T, \bar{\mu})$ is mixing and exhibits exponential decay of correlations for Hölder observables, and the Central Limit Theorem holds.

(6) More generally, the Fourier transform of the correlation function (sometimes called the power spectrum) admits a meromorphic extension in the annulus $\left\{z \in \mathbb{C} ; \tau<|z|<\tau^{-1}\right\}$, and the poles (Ruelle resonances) correspond exactly to the eigenvalues of $\mathcal{L}$.

Items (1)-(4) and part of (5) are proved in Section 5.2. The rest is proved in Section 5.3 .

Remark 2.9. Although $\bar{\mu}$ is a natural measure in the sense that it is obtained by pushing forward and averaging Lebesgue measure, it is generally not absolutely continuous with respect to Lebesgue in the hyperbolic setting. Typically, one expects 
$\bar{\mu}$ to be singular along stable manifolds and absolutely continuous along unstable manifolds.

Remark 2.10. A natural question is if all the positive elements of $\mathbb{V}_{0}$ are SRB measures; however, the characterization of SRB measures as measures that are absolutely continuous along unstable manifolds is a bit at odds with our philosophy since it would require us to prove the existence and properties of such manifolds in the first place. An alternative approach is to note that the integral along a manifold lying in the unstable cone yields an element of $\mathcal{B}$ (see GL, Proposition 4.4.] for a similar result in that context), and therefore by iterating it (one standard manner to construct SRB measures) one converges to the elements of $\mathbb{V}_{0}$. With this approach one can show that $\mathbb{V}_{0}$ corresponds exactly to the decomposition into SRB measures.

Remark 2.11. Several of the above results are similar to those obtained in $[\mathrm{P}, \mathrm{Y}]$ for piecewise hyperbolic maps. In [Y] an SRB measure $\nu$ was constructed, and under the assumption that $\left(T^{n}, \nu\right)$ is ergodic for all $n$, it was proven that $(T, \nu)$ satisfies the CLT and exponential decay of correlations for Hölder observables. In [P], the existence of SRB measures and the ergodic decomposition was proven.

In Section [6, we prove various perturbation results, using the framework provided by $[\mathrm{KL}$. This requires first obtaining uniform Lasota-Yorke estimates for the perturbed operators $\mathcal{L}_{\varepsilon}$. Then, regarding these operators as acting from $\mathcal{B}$ to $\mathcal{B}_{w}$, we define the norm

$$
\||\mathcal{L}|\|=\sup _{\{h \in \mathcal{B}:\|h\| \leq 1\}}|\mathcal{L} h|_{w}
$$

and show that $\mathcal{L}_{\varepsilon}$ and $\mathcal{L}$ are close in this norm. The results of $[\mathrm{KL}$ then imply that the spectral picture (hence the SRB measures, the rate of correlation decay, etc.) persists and is stable as long as a spectral gap is maintained. These results, to our knowledge, are new and are a simple byproduct of the present approach.

2.4. Deterministic and smooth random perturbations. We define the class of perturbations for which our results hold. This class is analogous to that studied in GL.

Fix $B_{*}<\infty$ such that $\left|D^{2} T\right|<B_{*}$ and let $\Gamma_{B_{*}}$ be the set of maps $\tilde{T}$ that satisfy the assumptions of Section 2 with $\left|D^{2} \tilde{T}\right| \leq B_{*}$.

Definition 1. Given two maps $T_{1}, T_{2} \in \Gamma_{B_{*}}$ we say that they have distance $\varepsilon$ if their singularity curves are at distance $\varepsilon$ and if outside an $\varepsilon$ neighborhood of the union of their singularity curves they are $\varepsilon$-close in the $\mathcal{C}^{2}$ norm. We call this distance $\gamma\left(T_{1}, T_{2}\right)$.

Choose $\varepsilon \leq \varepsilon_{0}$ and let $X_{\varepsilon}$ be an $\varepsilon$-neighborhood of $T$ in $\Gamma_{B_{*}}$,

$$
X_{\varepsilon}=\left\{\tilde{T} \in \Gamma_{B_{*}}: \gamma(T, \tilde{T})<\varepsilon\right\} .
$$

In general, the constants $\lambda(\tilde{T}), \mu(\tilde{T}), \mu_{+}(\tilde{T})$ and $D_{n}(\tilde{T})$ defined by (2.1) and (2.6) depend on the map $\tilde{T}$. However, for $\varepsilon \leq \varepsilon_{0}$, we may choose constants $\lambda, \mu, \mu_{+}$and $D_{n}$ such that $1<\lambda \leq \lambda(\tilde{T}), 1>\mu \geq \mu(\tilde{T}), 1>\mu_{+} \geq \mu(\tilde{T})$ and $D_{n} \geq D_{n}(\tilde{T})$ for all $\tilde{T} \in X_{\varepsilon}$. These are the constants we shall use in the estimates of Section 6 which enable us to obtain uniform Lasota-Yorke type inequalities for the maps in $X_{\varepsilon}$. 
Let $\nu$ be a probability measure on a probability space $\Omega$ and let $g: \Omega \times \mathcal{M} \rightarrow \mathbb{R}^{+}$ be a measurable function satisfying:

(i) $g(\omega, \cdot) \in \mathcal{C}^{1}\left(\mathcal{M}, \mathbb{R}^{+}\right)$for each $\omega \in \Omega$;

(ii) $\int_{\Omega} g(\omega, x) d \nu(\omega)=1$ for each $x \in \mathcal{M}$;

(iii) $g(\omega, x) \geq a>0$ and $|g(\omega, \cdot)|_{\mathcal{C}^{1}(\mathcal{M})} \leq A<\infty$.

If we associate to each $\omega \in \Omega$ a map $T_{\omega} \in X_{\varepsilon}$, this defines a random walk on $\mathcal{M}$ in a natural way. Starting at $x$, we choose $T_{\omega}$ according to the distribution $g(\omega, x) d \nu(\omega)$. We apply $T_{\omega}$ to $x$ and repeat this process starting at $T_{\omega} x$. We say the process has size $\Delta(\nu, g) \leq \varepsilon$.

Remark 2.12. If $\nu$ is a Dirac measure centered at $\omega_{0}$, this process corresponds to the deterministic perturbation $T_{\omega_{0}}$ of $T$. Thus this setting encompasses a large class of random and deterministic perturbations of $T$.

The transfer operator $\mathcal{L}_{\nu, g}$ associated with the random process governs the evolution of densities by

$$
\mathcal{L}_{\nu, g} h(x)=\int_{\Omega} \mathcal{L}_{T_{\omega}} h(x) g\left(\omega, T_{\omega}^{-1} x\right) d \nu(\omega)
$$

where $\mathcal{L}_{T_{\omega}}$ is the transfer operator associated with $T_{\omega}$.

Lemmas 6.1, 6.2 and 6.3 prove the two steps required in order to apply [KL] to the above class of perturbations. We need some more notation before stating the theorem fully.

Choose $\sigma \in\left(\max \left\{\lambda^{-\beta}, \rho, \mu_{+}^{q}\right\}, 1\right)$ and denote by $\operatorname{sp}(\mathcal{L})$ the spectrum of $\mathcal{L}$ on $\mathcal{B}$. Since $\operatorname{sp}(\mathcal{L}) \cap\{z \in \mathbb{C}:|z| \geq \sigma\}$ consists of a finite number of eigenvalues $\varrho_{1}, \ldots, \varrho_{k}$ of finite multiplicity, we may assume that $\operatorname{sp}(\mathcal{L}) \cap\{z \in \mathbb{C}:|z|=\sigma\}=\emptyset$. Hence there exists $t_{*}>0$ such that $\left|\varrho_{i}-\varrho_{j}\right|>t_{*}$ for $i \neq j$ and $\operatorname{dist}(\operatorname{sp}(\mathcal{L}),\{|z|=\sigma\})>t_{*}$.

Finally, for $t \leq t_{*}$, define the spectral projections

$$
\begin{aligned}
\Pi_{\nu, g}^{(j)} & :=\frac{1}{2 \pi i} \int_{\left|z-\varrho_{j}\right|=t}\left(z-\mathcal{L}_{\nu, g}\right)^{-1} d z \quad \text { and } \\
\Pi_{\nu, g}^{(\sigma)} & :=\frac{1}{2 \pi i} \int_{|z|=\sigma}\left(z-\mathcal{L}_{\nu, g}\right)^{-1} d z
\end{aligned}
$$

We denote by $\Pi_{0}^{(j)}$ and $\Pi_{0}^{(\sigma)}$ the corresponding spectral projections for the unperturbed operator $\mathcal{L}$.

Theorem 2.13. For each $t \leq t_{*}$ and $\eta<1-\frac{\log \sigma}{\log \max \left\{\lambda^{-\beta}, \rho, \mu_{+}^{q}\right\}}$, there exists $\varepsilon_{1}>$ 0 such that for any perturbation $(\nu, g)$ of $T$ satisfying $\Delta(\nu, g)<\varepsilon_{1}$, the spectral projections $\Pi_{0}^{(j)}, \Pi_{0}^{(\sigma)}, \Pi_{\nu, g}^{(j)}$ and $\Pi_{\nu, g}^{(\sigma)}$ are well-defined and satisfy

(1) $\left\|\left|\Pi_{\nu, g}^{(j)}-\Pi_{0}^{(j)}\right|\right\| \leq C \Delta(\nu, g)^{\eta}$ and $\left\|\mid \Pi_{\nu, g}^{(\sigma)}-\Pi_{0}^{(\sigma)}\right\| \| \leq C \Delta(\nu, g)^{\eta} ;$

(2) $\operatorname{rank}\left(\Pi_{\nu, g}^{(j)}\right)=\operatorname{rank}\left(\Pi_{0}^{(j)}\right)$ for each $j$;

(3) $\left\|\mathcal{L}_{\nu, g}^{n} \Pi_{\nu, g}^{(\sigma)}\right\| \leq C \sigma^{n}$ for all $n \geq 0$.

In view of the previous discussion on the meaning of the spectral data, Theorem 2.13 implies that the statistical properties (invariant measures, rates of decay of correlations, variance of the CLT, etc.) are stable under the above class of perturbations.

Remark 2.14. It is possible to obtain a constructive bound on $\varepsilon_{1}$ by estimating $\tau$ and using the bounds provided by $[\mathrm{KL}$. 
2.5. Hyperbolic systems with holes. Another interesting class of perturbations is the one obtained by opening small holes in the system, thus making it an open system from which particles or mass can escape. In such systems, we keep track of the iterates of points as long as they do not enter the holes.

Let $H \subset \mathcal{M}$ be an open set which we call the hole and define $\mathcal{M}^{0}=\mathcal{M} \backslash H$. Let $\mathcal{M}^{n}=\bigcap_{i=0}^{n} T^{i} \mathcal{M}^{0}$ be the set of points that has not escaped by time $n$. The map $\tilde{T}^{n}:=T^{n} \mid \mathcal{M}^{n}$ describes the dynamics in the presence of the hole, and the evolution of measures is described by the transfer operator

$$
\mathcal{L}_{H}^{n} h=\mathcal{L}^{n}\left(1_{\mathcal{M}^{n}} h\right) .
$$

Since $\tilde{T}$ is simply a restriction of $T$, the family of admissible leaves $\Sigma$ does not change. Let $r=\sup \{|W|: W \subset H, W \in \Sigma\}$, i.e. $r$ is the largest "diameter" of $H$ where length is measured along admissible leaves.

We make the following two assumptions on the hole:

(H1) $\quad H$ is comprised of a finite number of open, connected components whose boundaries consist of finitely many piecewise smooth curves. Moreover, for each smooth component $\omega$ of $\partial H$ and any point $x \in \omega$, either

(1) the tangent to $\omega$ at $x$ is bounded away from $C^{s}(x)$, or

(2) the curvature of $\omega$ at $x$ is greater than $B$ (in the definition of $\Xi$ from Section 3.1).

For any $W \in \Sigma$, let $P_{n}$ be the maximum number of connected components of $T^{-n} W \cap \bigcup_{i=0}^{n} T^{-i} H$.

(H2) There exists an integer $n_{1}>0$, such that $\left(\lambda \mu^{\alpha_{0}}\right)^{n_{1}}>P_{n_{1}}$.

Notice that we can iterate the inequality in (H2) by controlling $\delta$. For a fixed $N=k n_{1}$, we can choose $\delta$ so that $P_{k n_{1}} \leq P_{n_{1}}^{k}$. Thus we can make $P_{N}\left(\lambda \mu^{\alpha_{0}}\right)^{-N}$ as small as we like.

Convention 2.15. We will assume that $n_{1}=1$. If this is not the case, we can always consider a higher iterate of $T$ for which this is true once (H2) is satisfied. We refer to $P_{1}$ as simply $P$ and assume that $\lambda^{-1} \mu^{-\alpha_{0}}(L+P)<1$.

The observations following (H2) and (P1) imply that we can control $\lambda^{-n} \mu^{-\alpha n}\left(L_{n}+P_{n}\right)$ which is precisely what we need in order to prove the LasotaYorke inequalities for $\mathcal{L}_{H}$.

Remark 2.16. It is fairly easy to have holes that satisfy our assumptions: for example holes with boundaries transverse to the stable cones, convex holes with boundaries with curvature larger than $B$ or some appropriate mixture of the two. In the case of convex holes, $P=1$.

Remark 2.17. We do not distinguish between pieces of $\tilde{T}^{-n} W$ created by intersections with the hole and those created by the singularities of $T$. This is clear in the estimates of Sections 4 and 6.3 and justifies Remark 2.1 that all the theorems of Section 2.3 hold with the weaker conditions (H1) and (H2) on $\mathcal{S}^{-}$as long as we choose $\beta \leq \alpha / 2$. This restriction on $\beta$ stems from the observation that for strictly convex holes (singularities), two curves in $\Sigma$ which are $\varepsilon$-close to one another can differ in their intersection with the hole (singularity) by a length of at most $C \varepsilon^{1 / 2}$. (This is used in equation (6.12) of Section 6.3.) 
The spectral radius of $\mathcal{L}_{H}$ is typically $\vartheta<1$ when all the mass in the system eventually escapes. The analogous notion to an invariant measure in this setting is that of a conditionally invariant measure. For any Borel measure $\mu$, define $\tilde{T}_{*} \mu(A)=\mu\left(\tilde{T}^{-1} A\right)$ for any Borel set $A \subset \mathcal{M}$. A probability measure $\mu$ is called conditionally invariant with respect to $\tilde{T}$ if $\tilde{T}_{*} \mu=\lambda \mu$ for some $\lambda \leq 1$. It follows that $\lambda=\mu\left(\mathcal{M}^{1}\right)$ and that $-\log \lambda$ represents the exponential rate of escape from the system with respect to $\mu$.

In principle there can be many conditionally invariant measures with different eigenvalues; however, one can ask if there exists a natural conditionally invariant measure which is the forward limit of a reasonable class of measures under the nonlinear operator $\tilde{T}_{*}^{n} \mu /\left|\tilde{T}_{*}^{n} \mu\right|$ (see [DY] for a discussion of the issues involved). Lemma 6.5 and Proposition 6.6 place us in the setting of [KL and allow us to assert the following theorem.

Theorem 2.18. Let $H$ be a hole satisfying conditions (H1) and (H2) and choose $\beta \leq \alpha / 2$ in (2.4). Then for $\mathrm{Pr}^{\alpha}$ sufficiently small,

(1) The non-essential spectra and the relative spectral projectors of $\mathcal{L}$ and $\mathcal{L}_{H}$ outside the disk of radius $\tau$ are close in the sense of Theorem 2.13.

(2) If T has a unique SRB measure, then $\tilde{T}$ admits a unique natural conditionally invariant measure $\mu$ which is characterized by $\mu=\lim _{n \rightarrow \infty} \tilde{T}_{*}^{n} m /\left|\tilde{T}_{*}^{n} m\right|$.

Corollary 2.19. Suppose $T$ has a unique $S R B$ measure $\mu_{0}$ and let $H_{t}$ be a sequence of holes with diam $\left(H_{t}\right) \leq t$ satisfying (H1) and (H2) with uniform constant $n_{1}$. Let $\mu_{t}$ be the natural conditionally invariant measures associated with $H_{t}$ given by Theorem 2.18(2). Then $\left|\mu_{t}-\mu_{0}\right|_{w} \rightarrow 0$ as $t \rightarrow 0$.

Proof. The convergence follows directly from the closeness of the spectral projectors guaranteed by Theorem $2.18(1)$. Note that the convergence in the $|\cdot|_{w}$-norm is stronger than the weak-convergence results typically obtained for open systems.

When $T$ has a unique SRB measure, one can also associate to the conditionally invariant measure $\mu$ a unique invariant measure $\nu$ for $\tilde{T}$ which is supported on $\Omega=\bigcap_{n=-\infty}^{\infty} T^{n} \mathcal{M}^{0}$, the set of points that never escape from the system. Define $\Pi_{\vartheta}$ to be the projector onto the eigenspace associated with the spectral radius $\vartheta$. $\Pi_{\vartheta}$ admits the following characterization:

$$
\Pi_{\vartheta}=\lim _{n \rightarrow \infty} \vartheta^{-n} \mathcal{L}_{H}^{n} .
$$

In fact, the spectral decomposition implies that $\mathcal{L}_{H} h=\vartheta \mu \ell(h)+R h$, where the spectral radius of $R$ is strictly smaller than $\vartheta$ and

$$
\ell(h)=\int \Pi_{\vartheta} h d m=\lim _{n \rightarrow \infty} \vartheta^{-n} \int_{\mathcal{M}^{n}} h d m \quad \text { for } h \in \mathcal{B} .
$$

It is then easy to see that

$$
\nu(\varphi):=\ell(\varphi \mu)=\lim _{n \rightarrow \infty} \vartheta^{-n} \int_{\mathcal{M}^{n}} \varphi d \mu
$$

is the required invariant measure.

Remark 2.20. Hyperbolic systems with holes have been well-studied when the systems in question admit a finite Markov partition (see the long series of papers C, CM1, CM2, CMT1, CMT2, LM]), but these are the first results for hyperbolic systems with discontinuities and no Markov properties. Moreover, it should be 
noted that even if $T$ is a $\mathcal{C}^{2}$ Anosov diffeomorphism, then the present approach yields stronger results in a much more simple, direct and compact way than has previously been available. In one dimension, piecewise expanding maps with nonMarkov holes have been studied via a variety of approaches, [BC], LiM], D1]; logistic maps with non-Markov holes were studied in D2.

\section{BANACH SPACE EMBEDDINGS}

We must start with the overdue exact definition of the family of admissible leaves $\Sigma$, which is a set of parametrized curves in the unstable direction.

3.1. Family of admissible leaves. Our definitions are similar to those of GL].

For $\kappa$ sufficiently small, we redefine the stable cone at $x \in \mathcal{M}$ to be

$$
C^{s}(x)=\left\{u+v \in T_{x} M: u \in E^{s}(x), v \perp E^{s}(x),\|v\| \leq \kappa\|u\|\right\} .
$$

An analogous expression defines $C^{u}(x)$. These families of cones are invariant, that is, $D T^{-1}(x)\left(C^{s}(x)\right) \subset C^{s}\left(T^{-1} x\right)$ and $D T(x)\left(C^{u}(x)\right) \subset C^{u}(T x)$.

For each $\mathcal{M}_{i}^{+}$, we choose a finite number of coordinate charts $\left\{\chi_{j}\right\}_{j=1}^{K}$, whose domains $R_{j}$ vary depending on whether they contain a preimage of part of the boundary curves of $\mathcal{M}_{i}^{+}$. For those $\chi_{j}$ which map only to the interior of $\mathcal{M}_{i}^{+}$, we take $R_{j}=\left(-r_{j}, r_{j}\right)^{2}$. For those $\chi_{j}$ which map to a part of $\partial \mathcal{M}_{i}^{+}$, we take $R_{j}$ to be $\left(-r_{j}, r_{j}\right)^{2}$ restricted to one side of a piecewise $\mathcal{C}^{1}$ curve (the preimage of part of $\partial \mathcal{M}_{i}^{+}$) which we position so that it passes through the origin. Each $R_{j}$ has a centroid, $y_{j}$, and each $\chi_{j}$ satisfies

(1) $D \chi_{j}\left(y_{j}\right)$ is an isometry.

(2) $D \chi_{j}\left(y_{j}\right) \cdot(\mathbb{R} \times 0)=E^{s}\left(\chi_{j}\left(y_{j}\right)\right)$.

(3) The $\mathcal{C}^{2}$-norm of $\chi_{j}$ and its inverse are bounded by $1+\kappa$.

(4) There exists $c_{j} \in(\kappa, 2 \kappa)$ such that the cone $C_{j}=\left\{u+v \in \mathbb{R}^{2}: u \in\right.$ $\left.\mathbb{R} \times\{0\}, v \in\{0\} \times \mathbb{R},\|v\| \leq c_{j}\|u\|\right\}$ has the following property: for $x \in R_{j}$ such that $\chi_{j}(x) \notin \mathcal{S}^{-}, D \chi_{j}(x) C_{j} \supset C^{s}\left(\chi_{j}(x)\right)$ and $D T^{-1}\left(D \chi_{j}(x) C_{j}\right) \subset$ $C^{s}\left(T^{-1} \circ \chi_{j}(x)\right)$.

(5) $\mathcal{M}_{i}^{+}$is covered by the sets $\left\{\chi_{j}\left(R_{j} \cap\left(-\frac{r_{j}}{2}, \frac{r_{j}}{2}\right)^{2}\right)\right\}_{j=1}^{K}$.

Now choose $r_{0} \leq \min _{j} r_{j} / 2$; later, we may shrink $r_{0}$ further. Fix $B<\infty$ and consider the set of functions

$$
\Xi:=\left\{F \in \mathcal{C}^{2}([-r, r], \mathbb{R}): r \in\left(0, r_{0}\right], F(0)=0,|F|_{\mathcal{C}^{1}} \leq \kappa,|F|_{\mathcal{C}^{2}} \leq B\right\} .
$$

Let $I_{r}=(-r, r)$. For $x \in R_{j} \cap\left(-r_{j} / 2, r_{j} / 2\right)^{2}$ such that $x+(t, F(t)) \in R_{j}$ for $t \in I_{r}$, define $G(x, r, F)$ to be a lift of the graph of $F$ to $\mathcal{M}: G(x, r, F)(t):=$ $\chi_{j}(x+(t, F(t)))$ for $t \in I_{r}$. For ease of notation, we will often write $G_{F}$ for $G(x, r, F)$. We record here for future use that $\left|G_{F}\right|_{\mathcal{C}^{1}} \leq(1+\kappa)^{2}$ and $\left|G_{F}^{-1}\right|_{\mathcal{C}^{1}} \leq 1+\kappa$.

Our set of admissible leaves is then defined as follows:

$$
\Sigma:=\left\{W=G(x, r, F)\left(I_{r}\right): x \in R_{j} \cap\left(r_{j} / 2, r_{j} / 2\right)^{2}, r \leq r_{0}, F \in \Xi\right\} .
$$

If necessary, we shrink $r_{0}$ so that $\sup _{W \in \Sigma}|W| \leq 2 \delta$, where $\delta$ is the length scale referred to in the convention following property (P1).

We define an analogous family of approximate unstable leaves $\mathcal{F}^{u}$ which lie in the unstable cone $C^{u}$. 
For any two leaves $W_{1}\left(\chi_{i_{1}}, x_{1}, r_{1}, F_{1}\right)$ and $W_{2}\left(\chi_{i_{2}}, x_{2}, r_{2}, F_{2}\right)$ with $r_{1} \leq r_{2}$, we define the distance between them to be7

$$
d_{\Sigma}\left(W_{1}, W_{2}\right)=\eta\left(i_{1}, i_{2}\right)+\left|x_{1}-x_{2}\right|+\left|r_{1}-r_{2}\right|+2^{-1} B^{-1}\left|F_{1}-F_{2}\right|_{\mathcal{C}^{1}\left(I_{r_{1}}\right)}
$$

where $\eta(i, j)=0$ if $i=j$ and $\eta(i, j)=\infty$ otherwise, i.e., we can only compare leaves which are mapped under the same chart.

Given two functions $\varphi_{i} \in \mathcal{C}^{q}\left(W_{i}, \mathbb{C}\right)$, we define the distance between $\varphi_{1}, \varphi_{2}$ as

$$
d_{q}\left(\varphi_{1}, \varphi_{2}\right)=\left|\varphi_{1} \circ G_{F_{1}}-\varphi_{2} \circ G_{F_{2}}\right|_{\mathcal{C}^{q}\left(I_{r_{1}}, \mathbb{C}\right)} .
$$

3.2. Some technical facts. To understand the structure of the spaces $\mathcal{B}_{w}$ and $\mathcal{B}$ it is necessary to prove two preliminary results that will be needed in many other arguments throughout the paper. In particular we need some understanding of the properties of $T^{-n} W$ for $W \in \Sigma$. We use the distortion bounds of Appendix A throughout Sections 3 and 4 .

Let $\mathcal{W}_{0}=\{W\} \subset \Sigma$ and suppose we have defined $\mathcal{W}_{n-1} \subset \Sigma$. If $W^{\prime} \in \mathcal{W}_{n-1}$ contains any singularity points of $T^{-1}$, then $T^{-1} W^{\prime}$ is partitioned into at most $L$ pieces $W_{i}^{\prime}$, so that $T$ is smooth on each $W_{i}^{\prime}$. Next, if one of the components of $T^{-1} W^{\prime}$ has length greater than $2 \delta$, it is partitioned further into pieces of length between $\delta$ and $2 \delta$. We define $\mathcal{W}_{n}$ to be the collection of all pieces $W_{i} \subset T^{-n} W$ obtained in this way. It is a standard result of hyperbolic theory that each $W_{i}$ is in $\Sigma$ if $B$ is chosen sufficiently large in the definition of $\Sigma$.

Lemma 3.1. For any $0 \leq \varsigma \leq \alpha_{0}$ and each $W \in \Sigma$

$$
\left.\left.\sum_{W_{i} \in \mathcal{W}_{n}}\left|W_{i}\right|^{\varsigma}|| D T^{n}\right|^{-1} J_{W} T^{n}\right|_{\mathcal{C}^{0}\left(W_{i}\right)} \leq C \sum_{k=1}^{n} \delta^{\varsigma-1} \rho^{n-k} \int_{W}\left|D T^{-k}\right| d m+C|W|^{\varsigma} \rho^{n}
$$

where $J_{W} T^{n}$ denotes the Jacobian of $T^{n}$ along the leaf $T^{-n} W$.

Proof. For each $1 \leq k \leq n$, denote by $W_{i}^{k}$ the elements of $\mathcal{W}_{k}$. Let $A_{k}=\{i$ : $\left.\left|W_{i}^{k}\right|<\delta\right\}$ and $B_{k}=\left\{i:\left|W_{i}^{k}\right| \geq \delta\right\}$ denote the short and long pieces in $\mathcal{W}_{k}$, respectively. We regard $\left\{W_{i}^{k}\right\}_{i, k}$ as a tree with $W$ as its root and $\mathcal{W}_{k}$ as the $k^{\text {th }}$ level.

At level $n$, we collect the short pieces into groups as follows. Consider a piece $W_{i_{0}}^{n} \in \mathcal{W}_{n}$, not necessarily short. Let $W_{j}^{k}$ be the most recent long "ancestor" of $W_{i_{0}}^{n}$, i.e., $k=\max \left\{0 \leq m \leq n: T^{n-m}\left(W_{i_{0}}^{n}\right) \subset W_{j}^{m}\right.$ and $\left.j \in B_{m}\right\}$. If no such ancestor exists, set $k=0$ and $W_{j}^{k}=W$. Note that if $W_{i_{0}}^{n}$ is long, then $W_{j}^{k}=W_{i_{0}}^{n}$. Let

$$
J_{n}\left(W_{j}^{k}\right)=\left\{i: T^{n-k}\left(W_{i}^{n}\right) \subset W_{j}^{k} \text { and }\left|T^{\ell}\left(W_{i}^{n}\right)\right|<\delta \text { for } 0 \leq \ell \leq n-k-1\right\}
$$

be the set of indices corresponding to the short pieces which have the same most recent long ancestor as $W_{i_{0}}^{n}$, or the set $\left\{W_{i_{0}}^{n}\right\}$ if the piece is long. Since for any $i \in J_{n}\left(W_{j}^{k}\right),\left|T^{\ell}\left(W_{i}^{n}\right)\right|<\delta$ for all $0 \leq \ell \leq n-k-1$, we may estimate $\# J_{n}\left(W_{j}^{k}\right) \leq$ $L^{n-k}$ using Remark 2.3 and Convention 2.4. So using the distortion bounds given

\footnotetext{
${ }^{7}$ The reader can check that the triangle inequality holds in $\Sigma$.
} 
by equations (A.1) and (A.2), we estimate

$$
\begin{aligned}
& \left.\left.\sum_{i \in J_{n}\left(W_{j}^{k}\right)}\left|W_{i}^{n}\right|^{\varsigma}|| D T^{n}\right|^{-1} J_{W} T^{n}\right|_{\mathcal{C}^{0}\left(W_{i}^{n}\right)} \\
& \leq\left.\left. C \sum_{i \in J_{n}\left(W_{j}^{k}\right)}\left|T^{n-k} W_{i}^{n}\right|^{\varsigma}\left|\left(J_{W} T^{n-k}\right)^{1-\varsigma}\right| D T^{n-k}\right|^{-1}\right|_{\mathcal{C}^{0}\left(W_{i}^{n}\right)} \\
& \left.\left.\quad \cdot|| D T^{k}\right|^{-1} J_{W} T^{k}\right|_{C^{0}\left(W_{j}^{k}\right)} \\
& \leq\left.\left. C|| D T^{k}\right|^{-1} J_{W} T^{k}\right|_{C^{0}\left(W_{j}^{k}\right)}\left|W_{j}^{k}\right|^{\varsigma}\left(L^{1-\varsigma} \lambda^{-1} \mu^{-\varsigma}\right)^{n-k} \\
& \leq\left.\left. C|| D T^{k}\right|^{-1} J_{W} T^{k}\right|_{C^{0}\left(W_{j}^{k}\right)}\left|W_{j}^{k}\right|^{\varsigma} \rho^{n-k}
\end{aligned}
$$

where for the first inequality we have estimated $\left|W_{i}^{n}\right|\left|J_{W} T^{n-k}\right|_{\mathcal{C}^{0}\left(W_{i}^{n}\right)} \leq C\left|T^{n-k} W_{i}^{n}\right|$, and for the second we have used the Hölder inequality. Grouping all $i \in A_{n}$ in this way, we are left with estimates over long pieces only, so that using (3.1),

$$
\begin{aligned}
\left.\left.\sum_{i}\left|W_{i}^{n}\right|^{\varsigma}|| D T^{n}\right|^{-1} J_{W} T^{n}\right|_{\mathcal{C}^{0}\left(W_{i}^{n}\right)} & =\left.\left.\sum_{k=0}^{n} \sum_{j \in B_{k}} \sum_{i \in J_{n}\left(W_{j}^{k}\right)}\left|W_{i}^{n}\right|^{\varsigma}|| D T^{n}\right|^{-1} J_{W} T^{n}\right|_{\mathcal{C}^{0}\left(W_{i}^{n}\right)} \\
& \leq\left.\left. C \sum_{k=0}^{n} \sum_{j \in B_{k}}\left|W_{j}^{k}\right|^{\varsigma}|| D T^{k}\right|^{-1} J_{W} T^{k}\right|_{\mathcal{C}^{0}\left(W_{j}^{k}\right)} \rho^{n-k}
\end{aligned}
$$

For each $k \geq 1$, we have $\left|W_{j}^{k}\right| \geq \delta$ and $T^{k} W_{j_{1}}^{k} \cap T^{k} W_{j_{2}}^{k}=\emptyset$ if $j_{1} \neq j_{2}$. So we may sum over $j$, again using (A.1),

$$
\begin{aligned}
\left.\left.\sum_{j \in B_{k}}\left|W_{j}^{k}\right|^{\varsigma}|| D T^{k}\right|^{-1} J_{W} T^{k}\right|_{\mathcal{C}^{0}\left(W_{j}^{k}\right)} & \leq C \sum_{j \in B_{k}}\left|W_{j}^{k}\right|^{\varsigma-1} \int_{W_{j}^{k}}\left|D T^{k}\right|^{-1} J_{W} T^{k} d m \\
& \leq C \delta^{\varsigma-1} \int_{W}\left|D T^{-k}\right| d m
\end{aligned}
$$

Putting (3.3) together with (3.2), we conclude that

$$
\left.\left.\sum_{i}\left|W_{i}^{n}\right|^{\varsigma}|| D T^{n}\right|^{-1} J_{W} T^{n}\right|_{\mathcal{C}^{0}\left(W_{i}^{n}\right)} \leq C \sum_{k=1}^{n} \delta^{\varsigma-1} \rho^{n-k} \int_{W}\left|D T^{-k}\right| d m+C|W|^{\varsigma} \rho^{n}
$$

which proves the lemma.

As an immediate corollary of the above lemma we have

Lemma 3.2. For any $0 \leq \varsigma \leq \alpha$ and each $W \in \Sigma$

$$
\left.\left.\sum_{W_{i} \in \mathcal{W}_{n}}\left|W_{i}\right|^{\varsigma}|| D T^{n}\right|^{-1} J_{W} T^{n}\right|_{\mathcal{C}^{0}\left(W_{i}\right)} \leq C D_{n} \delta^{\varsigma-\alpha}|W|^{\alpha}+C|W|^{\varsigma} \rho^{n}
$$

Next, we have a fundamental lemma that will allow us to establish a connection between our Banach spaces and the standard spaces of distributions.

Lemma 3.3. For each $h \in \mathcal{C}^{1}(\mathcal{M}), n \geq 0$, and $\varphi \in \mathcal{C}_{\mathcal{S}_{n}^{+}}^{1}$ we have

$$
|h(\varphi)|=\left|\int_{\mathcal{M}} h \varphi d V\right| \leq C_{\delta}|h|_{w}\left(|\varphi|_{\infty}+\left|D^{s} \varphi\right|_{\infty}\right)
$$

where $D^{s}$ denotes the derivative along the stable direction and $d V$ is the normalized volume element on $\mathcal{M}$. 
Proof. Choose $\varphi \in \mathcal{C}_{\mathcal{S}_{n_{0}}^{+}}^{1}$ for some $n_{0} \in \mathbb{N}$, so that $\varphi \in \mathcal{C}^{1}(\bar{K})$ for each $K \in \mathcal{K}_{n_{0}}$. First partition each $\mathcal{M}_{i}^{+}$into finitely many approximate boxes $B_{\ell}$ whose boundary curves are elements of $\Sigma$ and $\mathcal{F}^{u}$, as well as the boundary curves of $\mathcal{M}_{i}^{+}$where necessary. The $B_{\ell}$ can be constructed so that each $B_{\ell}$ is foliated by curves $W \in \Sigma$ and $\operatorname{diam}\left(B_{\ell}\right) \leq 2 \delta$. On each $B_{\ell}$, choose a smooth partition $\left\{W_{\ell}(\xi)\right\}$ of $B_{\ell}$ made up of elements of $\Sigma$ which completely cross $B_{\ell}$ in the approximate stable direction. Here $\xi \in E_{\ell}$ is a parameter which indexes the elements of the foliation $\left\{W_{\ell}(\xi)\right\}$.

In order to integrate along the curves $W_{\ell}(\xi)$, we decompose the measure $d V$ on $B_{\ell}$ into $d V=\nu(d \xi) d m_{\xi, \ell}$, where $m_{\xi, \ell}$ is the conditional measure on the fiber $W_{\ell}(\xi)$ and $\nu$ is an appropriate measure on $\bigcup_{\ell} E_{\ell}$. We normalize the measures so that $m_{\xi, \ell}\left(W_{\ell}(\xi)\right)=\left|W_{\ell}(\xi)\right|$; thus, since the foliation is smooth, $d m_{\xi, \ell}=\rho_{\xi, \ell} d m$ where $m$ is the arc-length measure on $W_{\ell}(\xi)$ and $\rho_{\xi, \ell}, \rho_{\xi, \ell}^{-1} \in \mathcal{C}^{1}\left(W_{\ell}(\xi)\right)$. Note that $\nu\left(E_{\ell}\right)<\infty$.

Taking $n \geq n_{0}$, we estimate

$$
\begin{aligned}
\int_{\mathcal{M}} h \varphi d V & =\sum_{\ell} \int_{B_{\ell}} \mathcal{L}^{n} h \varphi \circ T^{-n} d V=\sum_{\ell} \int_{E_{\ell}} \nu(d \xi) \int_{W_{\ell}(\xi)} \mathcal{L}^{n} h \varphi \circ T^{-n} d m_{\xi, \ell} \\
& =\sum_{\ell} \int_{E_{\ell}} \nu(d \xi) \int_{W_{\ell}(\xi)} h \circ T^{-n}\left|D T^{n}\left(T^{-n}\right)\right|^{-1} \varphi \circ T^{-n} \rho_{\xi, \ell} d m
\end{aligned}
$$

where we have used the definition of $\mathcal{L}^{n} h$ in the second line. We estimate the integral by changing variables on one $W_{\ell}(\xi)$ at a time.

$$
\begin{gathered}
\int_{W_{\ell}(\xi)} \frac{h \circ T^{-n}}{\left|D T^{n}\left(T^{-n}\right)\right|} \varphi \circ T^{-n} \rho_{\xi, \ell} d m=\sum_{i} \int_{W_{\ell, i}^{n}(\xi)} h \varphi\left|D T^{n}\right|^{-1} J_{W} T^{n} \rho_{\xi, \ell} \circ T^{n} d m \\
\leq\left.\left. C|h|_{w} \sum_{i}|\varphi|_{\mathcal{C}^{1}\left(W_{\ell, i}^{n}(\xi)\right)}|| D T^{n}\right|^{-1} J_{W} T^{n}\right|_{\mathcal{C}^{1}\left(W_{\ell, i}^{n}(\xi)\right)}\left|\rho_{\xi, \ell} \circ T^{n}\right|_{\mathcal{C}^{1}\left(W_{\ell, i}^{n}(\xi)\right)}
\end{gathered}
$$

where $W_{\ell, i}^{n}(\xi)$ are the smooth components of $T^{-n} W_{\ell}(\xi)$ as defined earlier. Note that $\left|\rho_{\xi, \ell} \circ T^{n}\right|_{\mathcal{C}^{1}\left(W_{\ell, i}^{n}(\xi)\right)} \leq C\left|\rho_{\xi, \ell}\right|_{\mathcal{C}^{1}\left(W_{\ell}(\xi)\right)} \leq C$ for some $C$ independent of $\xi$ and $\ell$ using the estimate of equation (4.3) in Section 4.1. Also, the distortion bounds of Appendix A imply that $\left.\left.|| D T^{n}\right|^{-1} J_{W} T^{n}\right|_{\mathcal{C}^{1}\left(W_{\ell, i}^{n}(\xi)\right)} \leq\left.\left.|| D T^{n}\right|^{-1} J_{W} T^{n}\right|_{\mathcal{C}^{0}\left(W_{\ell, i}^{n}(\xi)\right)}$.

As $n$ increases, elements of $T^{-n} \Sigma$ become more closely aligned with the stable direction. So we may choose an $n_{1}$, depending on $\varphi$, but not on $\ell$ or $\xi$, such that for $n \geq n_{1}$ and each $i,|\varphi|_{\mathcal{C}^{1}\left(W_{\ell, i}^{n}(\xi)\right)} \leq 2\left(|\varphi|_{\infty}+\left|D^{s} \varphi\right|_{\infty}\right)$. For $n \geq n_{1}+n_{0}$, using (3.6), we estimate

$$
\int_{W_{\ell}(\xi)} \mathcal{L}^{n} h \varphi \circ T^{-n} d m_{\xi, \ell} \leq\left.\left. C|h|_{w}\left(|\varphi|_{\infty}+\left|D^{s} \varphi\right|_{\infty}\right) \sum_{i}|| D T^{n}\right|^{-1} J_{W} T^{n}\right|_{\mathcal{C}^{0}\left(W_{\ell, i}^{n}(\xi)\right)} .
$$

To estimate the sum in (3.7), we use Lemma 3.1, with $\varsigma=0$ :

$$
\left.\left.\sum_{i}|| D T^{n}\right|^{-1} J_{W} T^{n}\right|_{\mathcal{C}^{0}\left(W_{\ell}^{n}(\xi)\right)} \leq C \sum_{k=1}^{n} \delta^{-1} \rho^{n-k} \int_{W}\left|D T^{-k}\right| d m+C \rho^{n} .
$$


This, together with (3.7), allows us to estimate (3.5):

$$
\begin{aligned}
& \int_{\mathcal{M}} h \varphi d V \leq C|h|_{w}\left(|\varphi|_{\infty}+\left|D^{s} \varphi\right|_{\infty}\right) \\
& \cdot\left(\sum_{\ell} \int_{E_{\ell}} \nu(d \xi) \rho^{n}+\delta^{-1} \sum_{k=1}^{n} \int_{\mathcal{M}}\left|D T^{-k}\right| d V \rho^{n-k}\right) .
\end{aligned}
$$

Since the integral $\int_{\mathcal{M}}\left|D T^{-k}\right| d V=1$ for each $k$, the sum over $k \geq 1$ is bounded independently of $n$. This proves the lemma.

3.3. Embeddings and compactness. Notice that, by definition, $|\cdot|_{w} \leq C\|\cdot\|_{s}$. This means that there exists a natural embedding of $\mathcal{B}$ into $\mathcal{B}_{w}$. In addition, if $h \in \mathcal{B}$ and $|h|_{w}=0$, it is immediate from the definitions (2.2), (2.3) and (2.4) that $\|h\|=0$, i.e. that the embedding is injective. Accordingly, we will consider $\mathcal{B}$ as a subset of $\mathcal{B}_{w}$ in what follows.

Remark 3.4. Lemma 3.3 implies that, for each $h \in \mathcal{B}_{w}$ and $\varphi \in \mathcal{C}_{\mathcal{S}_{n}^{+}}^{1},|h(\varphi)| \leq$ $C|h|_{w}|\varphi|_{\mathcal{C}_{\mathcal{S}_{n}^{+}}^{1}}$, that is, $\mathcal{C}^{1} \hookrightarrow \mathcal{B} \hookrightarrow \mathcal{B}_{w} \hookrightarrow\left(\mathcal{C}_{\mathcal{S}_{n}^{+}}^{1}\right)^{\prime}$. In fact, the inclusions are injective: if $h_{1}, h_{2}$ coincide as elements of $\left(\mathcal{C}_{\mathcal{S}_{n}^{+}}^{1}\right)^{\prime}$ and they both belong to any of the spaces $\mathcal{C}^{1}, \mathcal{B}$, or $\mathcal{B}_{w}$, then they coincide as elements of those spaces as well. This can be proven as in GL, Proposition 4.1].

We can finally state the last result of this section.

Lemma 3.5. The unit ball of $\mathcal{B}$ is compactly embedded in $\mathcal{B}_{w}$.

To prove the above fact it is convenient to remark the following obvious result.

Lemma 3.6. For any fixed $W \in \Sigma$, the unit ball of $|\cdot|_{\mathcal{C}^{1}(W)}$ is compactly embedded in $|\cdot|_{W, \alpha, q}$.

Proof. For fixed $W,|\cdot|_{W, \alpha, q}$ is equivalent to $|\cdot|_{\mathcal{C}^{q}(W)}$. The lemma follows immediately.

Proof of Lemma 3.5. Since on each leaf $W \in \Sigma,\|\cdot\|_{s}$ is the dual of $|\cdot|_{W, \alpha, q}$ and $|\cdot|_{w}$ is the dual of $|\cdot|_{\mathcal{C}^{1}(W)}$, Lemma 3.6 implies that the unit ball of $\|\cdot\|_{s}$ is compactly embedded in $|\cdot|_{w}$ on $W$. It remains to compare the weak norm on different leaves.

Let $0<\varepsilon \leq \varepsilon_{0}$ be fixed. The set of functions $\Xi$ is compact in the $\mathcal{C}^{1}$-norm, so on each $\mathcal{M}_{i}^{+}$we may choose finitely many leaves $W^{i} \in \Sigma$ such that $\left\{W^{i}\right\}$ forms an $\varepsilon$-covering of $\left.\Sigma\right|_{\mathcal{M}_{i}}$ in the distance $d_{\Sigma}$. Since any ball of finite radius in the $\mathcal{C}^{1}$-norm is compactly embedded in $\mathcal{C}^{q}$, we may choose finitely many functions $\bar{\varphi}_{j} \in \mathcal{C}^{1}\left(I_{r_{0}}\right)$ such that $\left\{\bar{\varphi}_{j}\right\}$ forms an $\varepsilon$-covering in the $\mathcal{C}^{q}\left(I_{r_{0}}\right)$-norm of the ball of radius $(1+\kappa)^{2}$ in $\mathcal{C}^{1}\left(I_{r_{0}}\right)$.

Now let $h \in \mathcal{C}^{1}(\mathcal{M}), W \in \Sigma$, and $\varphi \in \mathcal{C}^{1}(W)$ with $|\varphi|_{\mathcal{C}^{1}(W)} \leq 1$. Let $F$ denote the function associated with $W$ and as usual, let $G_{F}$ be the lift of the graph of $F$ to $\mathcal{M}$. Let $\bar{\varphi}=\varphi \circ G_{F}$ be the push down of $\varphi$ to $I_{r}$. Note that $|\bar{\varphi}|_{\mathcal{C}^{1}\left(I_{r}\right)} \leq(1+\kappa)^{2}$.

Choose $W^{i}$ such that $d_{\Sigma}\left(W, W^{i}\right) \leq \varepsilon$ and $\bar{\varphi}_{j}$ such that $\left|\bar{\varphi}-\bar{\varphi}_{j}\right|_{\mathcal{C}^{q}\left(I_{r}\right)} \leq \varepsilon$. Let $F^{i}$ and $G_{F^{i}}$ denote the usual functions associated with the leaf $W^{i}$ and define $\varphi_{j}=\bar{\varphi}_{j} \circ G_{F^{i}}^{-1}$. Note that $\left|\varphi_{j}\right|_{\mathcal{C}^{1}\left(W^{i}\right)} \leq(1+\kappa)^{3}$. Then normalizing $\varphi$ and $\varphi_{j}$ by $(1+\kappa)^{3}$, we get

$$
\left|\int_{W} h \varphi d m-\int_{W^{i}} h \varphi_{j} d m\right| \leq \varepsilon^{\beta}\|h\|_{u}(1+\kappa)^{3} \leq \varepsilon^{\beta}(1+\kappa)^{3} b^{-1}\|h\| .
$$


We have proved that for each $0<\varepsilon \leq \varepsilon_{0}$, there exist finitely many bounded linear functionals $\ell_{i, j}, \ell_{i, j}(h)=\int_{W^{i}} h \varphi_{j} d m$, such that

$$
|h|_{w} \leq \sup _{i, j} \ell_{i, j}(h)+\varepsilon^{\beta} C_{b}\|h\|,
$$

which implies the desired compactness.

\section{LAsota-YoRKe estimates}

In this section we prove Proposition 2.7.

4.1. Estimating the weak norm. For $h \in \mathcal{C}^{1}(\mathcal{M}), W \in \Sigma$ and $\varphi \in \mathcal{C}^{1}(W)$ such that $|\varphi|_{\mathcal{C}^{1}(W)} \leq 1$, we have

$$
\int_{W} \mathcal{L}^{n} h \varphi d m=\int_{T^{-n} W} h \frac{J_{W} T^{n}}{\left|D T^{n}\right|} \varphi \circ T^{n} d m=\sum_{W_{i} \in \mathcal{W}_{n}} \int_{W_{i}} h \frac{J_{W} T^{n}}{\left|D T^{n}\right|} \varphi \circ T^{n} d m
$$

where as before $J_{W} T^{n}$ denotes the Jacobian of $T^{n}$ along the leaf $T^{-n} W$.

Using the definition of the weak norm on each $W_{i}$, we estimate (4.1) by

$$
\int_{W} \mathcal{L}^{n} h \varphi d m \leq\left.\left.\sum_{W_{i} \in \mathcal{W}_{n}}|h|_{w}|| D T^{n}\right|^{-1} J_{W} T^{n}\right|_{\mathcal{C}^{1}\left(W_{i}\right)}\left|\varphi \circ T^{n}\right|_{\mathcal{C}^{1}\left(W_{i}\right)}
$$

The disortion bounds given by equation (A.1) imply that

$$
\left.\left.|| D T^{n}\right|^{-1} J_{W} T^{n}\right|_{\mathcal{C}^{1}\left(W_{i}\right)} \leq\left.\left. C|| D T^{n}\right|^{-1} J_{W} T^{n}\right|_{\mathcal{C}^{0}\left(W_{i}\right)} .
$$

Also notice that

$$
\frac{\left|\varphi\left(T^{n} x\right)-\varphi\left(T^{n} y\right)\right|}{d_{s}\left(T^{n} x, T^{n} y\right)} \cdot \frac{d_{s}\left(T^{n} x, T^{n} y\right)}{d_{s}(x, y)} \leq C|\varphi|_{\mathcal{C}^{1}(W)}\left|J_{W} T^{n}\right|_{\mathcal{C}^{0}\left(W_{i}\right)} \leq C \lambda^{-n}|\varphi|_{\mathcal{C}^{1}(W)}
$$

for any $x, y \in W_{i}$, so that $\left|\varphi \circ T^{n}\right|_{\mathcal{C}^{1}\left(W_{i}\right)} \leq C|\varphi|_{\mathcal{C}^{1}(W)}$. Using these estimates in equation (4.2), we obtain

$$
\int_{W} \mathcal{L}^{n} h \varphi d m \leq\left.\left. C|h|_{w}|\varphi|_{\mathcal{C}^{1}(W)} \sum_{W_{i} \in \mathcal{W}_{n}}|| D T^{n}\right|^{-1} J_{W} T^{n}\right|_{\mathcal{C}^{0}\left(W_{i}\right)} .
$$

The above formula, together with Lemma 3.2 used in the case $\varsigma=0$, yields the inequality

$$
\int_{W} \mathcal{L}^{n} h \varphi d m \leq C|h|_{w}\left(D_{n}+\rho^{n}\right)|\varphi|_{\mathcal{C}^{1}(W)} .
$$

Taking the supremum over all $W \in \Sigma$ and $\varphi \in \mathcal{C}^{1}(W)$ with $|\varphi|_{\mathcal{C}^{1}(W)} \leq 1$ yields the required estimate (2.7).

4.2. Estimating the strong stable norm. Using equation (4.1), we write for each $W \in \Sigma$ and $\varphi \in \mathcal{C}^{1}(W, \mathbb{C})$ such that $|\varphi|_{W, \alpha, q} \leq 1$,

$$
\int_{W} \mathcal{L}^{n} h \varphi d m=\sum_{i}\left\{\int_{W_{i}} h \frac{J_{W} T^{n}}{\left|D T^{n}\right|} \bar{\varphi}_{i}+\frac{1}{\left|W_{i}\right|} \int_{W_{i}} \varphi \circ T^{n} \int_{W_{i}} h \frac{J_{W} T^{n}}{\left|D T^{n}\right|}\right\},
$$

where $\bar{\varphi}_{i}:=\varphi \circ T^{n}-\frac{1}{\left|W_{i}\right|} \int_{W_{i}} \varphi \circ T^{n} d m$. Let us estimate the above expression. 
To estimate the first term of (4.4), we first estimate $\left|\bar{\varphi}_{i}\right|_{\mathcal{C}^{q}\left(W_{i}\right)}$. Following equation (4.3), we write

$$
\frac{\left|\varphi\left(T^{n} x\right)-\varphi\left(T^{n} y\right)\right|}{d_{s}(x, y)^{q}} \leq C\left|J_{W} T^{n}\right|_{\mathcal{C}^{1}\left(W_{i}\right)}^{q}|\varphi|_{\mathcal{C}^{1}(W)}
$$

for any $x, y \in W_{i}$. If $H^{q}(f)$ represents the Hölder constant of $f$, then (4.5) implies that $H^{q}\left(\varphi \circ T^{n}\right) \leq C\left|J_{W} T^{n}\right|_{\mathcal{C}^{0}\left(W_{i}\right)}^{q} H^{q}(\varphi)$ on approximate stable leaves due to the contraction of $T^{n}$. Also,

$$
\begin{gathered}
\left|\varphi \circ T^{n}-\frac{1}{\left|W_{i}\right|} \int_{W_{i}} \varphi \circ T^{n} d m\right|_{\mathcal{C}^{0}\left(W_{i}\right)} \leq\left|\sup _{W_{i}} \varphi \circ T^{n}-\inf _{W_{i}} \varphi \circ T^{n}\right| \\
\leq H^{q}\left(\varphi \circ T^{n}\right)\left|W_{i}\right|^{q} \leq C H^{q}(\varphi)\left|J_{W} T^{n}\right|_{\mathcal{C}^{0}\left(W_{i}\right)}^{q} .
\end{gathered}
$$

This estimate together with (4.5) and the fact that $|\varphi|_{W, \alpha, q} \leq 1$, implies

$$
\left|\bar{\varphi}_{i}\right|_{\mathcal{C}^{q}\left(W_{i}\right)} \leq C\left|J_{W} T^{n}\right|_{\mathcal{C}^{0}\left(W_{i}\right)}^{q}|\varphi|_{\mathcal{C}^{q}(W)} \leq C\left|J_{W} T^{n}\right|_{\mathcal{C}^{0}\left(W_{i}\right)}^{q}|W|^{-\alpha} .
$$

Applying (4.6) and the definition of the strong stable norm to the first term of (4.4) yields

$$
\begin{gathered}
\sum_{i} \int_{W_{i}} h \frac{J_{W} T^{n}}{\left|D T^{n}\right|} \bar{\varphi}_{i} d m \leq\left.\left. C \sum_{i}\|h\|_{s}\left|W_{i}\right|^{\alpha}|| D T^{n}\right|^{-1} J_{W} T^{n}\right|_{\mathcal{C}^{0}\left(W_{i}\right)}\left|J_{W} T^{n}\right|_{\mathcal{C}^{0}\left(W_{i}\right)}^{q}|W|^{-\alpha} \\
\leq\left.\left. C\|h\|_{s}|W|^{-\alpha} \mu_{+}^{q n} \sum_{i}\left|W_{i}\right|^{\alpha}|| D T^{n}\right|^{-1} J_{W} T^{n}\right|_{\mathcal{C}^{0}\left(W_{i}\right)} \leq C D_{n}\|h\|_{s} \mu_{+}^{q n}
\end{gathered}
$$

where in the second line we have used Lemma 3.2 with $\varsigma=\alpha$.

For the second term of (4.4), we use the fact that $|\varphi|_{\infty} \leq|W|^{-\alpha}$ to estimate $\frac{1}{\left|W_{i}\right|} \int_{W_{i}} \varphi \circ T^{n} d m \leq|W|^{-\alpha}$. Recall the notation used in the proof of Lemma 3.1 Grouping the pieces $W_{i}=W_{i}^{n}$ according to most recent long ancestors, we have

$$
\begin{aligned}
\sum_{i}|W|^{-\alpha} \int_{W_{i}} h\left|D T^{n}\right|^{-1} J_{W} T^{n} d m \\
=\sum_{k=1}^{n} \sum_{j \in B_{k}} \sum_{i \in J_{n}\left(W_{j}^{k}\right)}|W|^{-\alpha} \int_{W_{i}} h\left|D T^{n}\right|^{-1} J_{W} T^{n} d m \\
\quad+\sum_{i \in J_{n}\left(W_{j}^{0}\right)}|W|^{-\alpha} \int_{W_{i}} h\left|D T^{n}\right|^{-1} J_{W} T^{n} d m
\end{aligned}
$$

where we have split up the terms involving $k=0$ and $k \geq 1$. We estimate the terms with $k \geq 1$ by the weak norm and the terms with $k=0$ by the strong stable norm,

$$
\begin{aligned}
\left.\sum_{i}|W|^{-\alpha}\left|\int_{W_{i}} h\right| D T^{n}\right|^{-1} J_{W} T^{n} d m \mid \\
\leq\left.\left. C \sum_{k=1}^{n} \sum_{j \in B_{k}} \sum_{i \in J_{n}\left(W_{j}^{k}\right)}|W|^{-\alpha}|h|_{w}|| D T^{n}\right|^{-1} J_{W} T^{n}\right|_{\mathcal{C}^{0}\left(W_{i}\right)} \\
\quad+\left.\left.C \sum_{i \in J_{n}\left(W_{j}^{0}\right)}|W|^{-\alpha}\|h\|_{s}\left|W_{i}\right|^{\alpha}|| D T^{n}\right|^{-1} J_{W} T^{n}\right|_{\mathcal{C}^{0}\left(W_{i}\right)} .
\end{aligned}
$$


Using equations (3.3) and (3.4) from Lemma 3.1, with $\varsigma=0$ for the first sum and $\varsigma=\alpha$ for the second, we conclude that

$$
\left.\left.\sum_{i} \frac{1}{\left|W_{i}\right|}\left|\int_{W_{i}} \varphi \circ T^{n} d m \int_{W_{i}} h\right| D T^{n}\right|^{-1} J_{W} T^{n} d m\left|\leq C D_{n} \delta^{-\alpha}\right| h\right|_{w}+C\|h\|_{s} \rho^{n} .
$$

Putting together (4.7) and (4.8) proves (2.8):

$$
\left\|\mathcal{L}^{n} h\right\|_{s} \leq C\left(D_{n} \mu_{+}^{q n}+\rho^{n}\right)\|h\|_{s}+C_{\delta} D_{n}|h|_{w} .
$$

4.3. Estimating the strong unstable norm. Consider two admissible leaves $W^{i} \in \Sigma, d_{\Sigma}\left(W^{1}, W^{2}\right) \leq \varepsilon$. They can be partitioned into "matched" pieces $U_{j}^{i}$ and "unmatched" pieces $V_{j}^{i}$. To do so consider the connected pieces of $W^{i} \backslash \mathcal{S}_{n}^{-}$. If one looks at their image under $T^{-n}$, then one can associate to each point $x \in$ $T^{-n}\left(W^{1} \cup W^{2}\right)$ a vertical (in the chart) segment $\gamma_{x} \in \mathcal{F}^{u}$, of length at most $C \lambda^{-n} \varepsilon$, such that its image under $T^{n}$, if not cut by a singularity, will be of length $C \varepsilon$ centered at $x$. We can thus subdivide the connected pieces of $W^{i} \backslash \mathcal{S}_{n}^{-}$into subintervals of points for which $T^{n} \gamma_{x}$ intersects the other manifold and subintervals for which this is not the case. In the latter case, we call the subintervals $V_{j}^{i}$ and note that either we are at the endpoints of $W^{i}$ or the vertical segment is cut by a singularity. In both cases the subintervals $V_{j}^{i}$ can be of length at most $C \varepsilon$ and their number is at most $L_{n}+28$ In the remaining pieces the curves $T^{n} \gamma_{x}$ provide a one to one correspondence between points in $W^{1}$ and $W^{2}$. We can further partition the pieces in such a way that the lengths of their preimages are between $\delta$ and $2 \delta$ and the partitioning can be made so that the pieces are pairwise matched by the foliation $\left\{\gamma_{x}\right\}$. We call these matched pieces $U_{j}^{i}$. In this way we write $W^{i}=\left(\bigcup_{j} U_{j}^{i}\right) \cup\left(\bigcup_{k} V_{k}^{i}\right)$. Note that the unmatched pieces $V_{j}^{i}$ must be short while the matched pieces $U_{j}^{i}$ may be long or short.

To be more precise, remember that to exactly describe the leaf $T^{-n} U_{j}^{1}$ we must specify $i_{j}, x_{j}, r_{j}, F_{j}^{1}$ so that $T^{-n} U_{j}^{1}=\chi_{i_{j}}\left(G\left(x_{j}, r_{j}, F_{j}^{1}\right)\left(I_{r_{j}}\right)\right)$ (see the end of Section 3.1). Once the leaves $T^{-n} U_{j}^{1}$ are described in such a way we have, by construction, that $T^{-n} U_{j}^{2}$ is of the form $G\left(x_{j}, r_{j}, F_{j}^{2}\right)\left(I_{r_{j}}\right)$ for some appropriate function $F_{j}^{2}$ so that the point $z:=x_{j}+\left(t, F_{j}^{1}(t)\right)$ is associated with the point $x_{j}+\left(t, F_{j}^{2}(t)\right) \in$ $\chi_{i_{j}}^{-1}\left(T^{-n} U_{j}^{2}\right)$ by the vertical segment $\chi_{i_{j}}^{-1}\left(\gamma_{\chi_{i_{j}}(z)}\right)=\{(0, s)\}_{s \in \mathbb{R}}$.

Given $\varphi_{i}$ on $W^{i}$ with $\left|\varphi_{i}\right|_{\mathcal{C}^{1}\left(W^{i}\right)} \leq 1$ and $d_{q}\left(\varphi_{1}, \varphi_{2}\right) \leq \varepsilon$, with the above construction we must estimate

$$
\begin{aligned}
\mid \int_{W^{1}} \mathcal{L}^{n} h \varphi_{1} d m & -\int_{W^{2}} \mathcal{L}^{n} h \varphi_{2} d m\left|\leq \sum_{i, j}\right| \int_{T^{-n} V_{j}^{i}} h\left|D T^{n}\right|^{-1} J_{W^{i}} T^{n} \varphi_{i} \circ T^{n} d m \mid \\
+ & \left.\sum_{j}\left|\int_{T^{-n} U_{j}^{1}} h\right| D T^{n}\right|^{-1} J_{W^{1}} T^{n} \varphi_{1} \circ T^{n} d m \\
& -\int_{T^{-n} U_{j}^{2}} h\left|D T^{n}\right|^{-1} J_{W^{2}} T^{n} \varphi_{2} \circ T^{n} d m \mid .
\end{aligned}
$$

${ }^{8}$ Without any loss of information (by throwing out at most finitely many points), we can take each $V_{j}^{i}$ to be the image of an open interval. Thus for fixed $i$, the $V_{j}^{i}$ are disjoint. 
We do the estimate over the unmatched short pieces $V_{j}^{i}$ first. Using the strong stable norm, we can compute

$$
\begin{aligned}
& \left.\sum_{i, j}\left|\int_{T^{-n} V_{j}^{i}} h\right| D T^{n}\right|^{-1} J_{W} T^{n} \varphi_{i} \circ T^{n} d m \mid \\
& \quad \leq\left.\left.\sum_{i, j}\|h\|_{s}\left|T^{-n} V_{j}^{i}\right|^{\alpha}|| D T^{n}\right|^{-1} J_{W} T^{n}\right|_{\mathcal{C}^{q}}\left|\varphi_{i}\right|_{\mathcal{C}^{q}} \\
& \quad \leq\left.\left. C\|h\|_{s} \sum_{i, j}\left|V_{j}^{i}\right|^{\alpha}|| D T^{n}\right|^{-1}\left(J_{W} T^{n}\right)^{1-\alpha}\right|_{\mathcal{C}^{0}} \leq C \varepsilon^{\alpha}\|h\|_{s} L_{n} \lambda^{-n} \mu^{-\alpha n} .
\end{aligned}
$$

Next, we must estimate

$$
\left.\sum_{j}\left|\int_{T^{-n} U_{j}^{1}} h\right| D T^{n}\right|^{-1} J_{W^{1}} T^{n} \varphi_{1} \circ T^{n} d m-\int_{T^{-n} U_{j}^{2}} h\left|D T^{n}\right|^{-1} J_{W^{2}} T^{n} \varphi_{2} \circ T^{n} d m \mid .
$$

First, recall that for each $F \in \Xi, G_{F}(t)=\chi\left(x_{F}+(t, F(t))\right)$ for $t \in I_{r}$. We define the map $\Psi: U_{j}^{2} \rightarrow U_{j}^{1}$ by $\Psi:=T^{n} \circ G_{F_{j}^{1}} \circ G_{F_{j}^{2}}^{-1} \circ T^{-n}$ and the function

$$
\tilde{\varphi}:=\left[\varphi_{1} \cdot\left(\left|D T^{n}\right|^{-1} J_{W^{1}} T^{n}\right) \circ T^{-n}\right] \circ \Psi \cdot\left[\left(\left|D T^{n}\right|^{-1} J_{W^{2}} T^{n}\right) \circ T^{-n}\right]^{-1} .
$$

$\tilde{\varphi}$ is well-defined on $U_{j}^{2}$ and

$$
\left[\tilde{\varphi} \circ T^{n}\left|D T^{n}\right|^{-1} J_{W^{2}} T^{n}\right] \circ G_{F_{j}^{2}}=\left[\varphi_{1} \circ T^{n}\left|D T^{n}\right|^{-1} J_{W^{1}} T^{n}\right] \circ G_{F_{j}^{1}} .
$$

We can then write

$$
\begin{aligned}
& \left.\sum_{j}\left|\int_{T^{-n} U_{j}^{1}} h\right| D T^{n}\right|^{-1} J_{W^{1}} T^{n} \varphi_{1} \circ T^{n}-\int_{T^{-n} U_{j}^{2}} h\left|D T^{n}\right|^{-1} J_{W^{2}} T^{n} \varphi_{2} \circ T^{n} \mid \\
& \leq\left.\sum_{j}\left|\int_{T^{-n} U_{j}^{1}} h\right| D T^{n}\right|^{-1} J_{W^{1}} T^{n} \varphi_{1} \circ T^{n}-\int_{T^{-n} U_{j}^{2}} h\left|D T^{n}\right|^{-1} J_{W^{2}} T^{n} \tilde{\varphi} \circ T^{n} \mid \\
& \quad+\left.\sum_{j}\left|\int_{T^{-n} U_{j}^{2}} h\right| D T^{n}\right|^{-1} J_{W^{2}} T^{n}\left(\tilde{\varphi}-\varphi_{2}\right) \circ T^{n} \mid .
\end{aligned}
$$

We estimate the first sum in equation (4.11) using the strong unstable norm.

The distortion bounds given by (A.1) and the estimate of (4.3) imply that (4.12)

$$
\left.\left.|| D T^{n}\right|^{-1} J_{W^{1}} T^{n} \cdot \varphi_{1} \circ T^{n}\right|_{\mathcal{C}^{1}\left(T^{-n} U_{j}^{1}\right)} \leq\left.\left. C\left|\varphi_{1}\right|_{\mathcal{C}^{1}\left(U_{j}^{1}\right)}|| D T^{n}\right|^{-1} J_{W^{1}} T^{n}\right|_{\mathcal{C}^{0}\left(T^{-n} U_{j}^{1}\right)} .
$$

Also, by the definition of $\tilde{\varphi}$,

$$
\begin{aligned}
& \left|\frac{J_{W^{2}} T^{n}}{\left|D T^{n}\right|} \tilde{\varphi} \circ T^{n}\right|_{\mathcal{C}^{1}\left(T^{-n} U_{j}^{2}\right)}=\left|\left[\varphi_{1} \circ T^{n} \cdot\left(\left|D T^{n}\right|^{-1} J_{W^{1}} T^{n}\right)\right] \circ G_{F_{j}^{1}} \circ G_{F_{j}^{2}}^{-1}\right|_{\mathcal{C}^{1}\left(T^{-n} U_{j}^{2}\right)} \\
& \quad \leq\left.\left. C\left|\varphi_{1}\right|_{\mathcal{C}^{1}\left(U_{j}^{1}\right)}|| D T^{n}\right|^{-1} J_{W^{1}} T^{n}\right|_{\mathcal{C}^{0}\left(T^{-n} U_{j}^{1}\right)} .
\end{aligned}
$$

By the definition of $\tilde{\varphi}$ and $d_{q}$,

$$
\begin{aligned}
& d_{q}\left(\left|D T^{n}\right|^{-1} J_{W^{2}} T^{n} \tilde{\varphi} \circ T^{n},\left|D T^{n}\right|^{-1} J_{W^{1}} T^{n} \varphi_{1} \circ T^{n}\right) \\
& \quad=\left|\left[\left|D T^{n}\right|^{-1} J_{W^{2}} T^{n} \tilde{\varphi} \circ T^{n}\right] \circ G_{F_{j}^{2}}-\left[\left|D T^{n}\right|^{-1} J_{W^{1}} T^{n} \varphi_{1} \circ T^{n}\right] \circ G_{F_{j}^{1}}\right|=0 .
\end{aligned}
$$


In addition, the uniform hyperbolicity of $T$ implies that

$$
d_{\Sigma}\left(T^{-n} U_{j}^{1}, T^{-n} U_{j}^{2}\right) \leq C \lambda^{-n} \varepsilon=: \varepsilon_{1} .
$$

This follows from the usual graph transform argument which is standard to hyperbolic theory.

We first renormalize the test functions by

$$
R_{j}=\left.\left.C\left|\varphi_{1}\right|_{\mathcal{C}^{1}}|| D T^{n}\right|^{-1} J_{W^{1}} T^{n}\right|_{\mathcal{C}^{0}\left(T^{-n} U_{j}^{1}\right)}
$$

Then for each $j$, we apply the definition of the strong unstable norm with $\varepsilon_{1}$ in place of $\varepsilon$. Thus,

$$
\begin{aligned}
& \left.\sum_{j}\left|\int_{T^{-n} U_{j}^{1}} h\right| D T^{n}\right|^{-1} J_{W^{1}} T^{n} \varphi_{1} \circ T^{n}-\int_{T^{-n} U_{j}^{2}} h\left|D T^{n}\right|^{-1} J_{W^{2}} T^{n} \tilde{\varphi} \circ T^{n} \mid \\
& \quad \leq\left.\left. C \varepsilon_{1}^{\beta} \sum_{j}\left|\varphi_{1}\right|_{\mathcal{C}^{1}}|| D T^{n}\right|^{-1} J_{W^{1}} T^{n}\right|_{\mathcal{C}^{0}\left(T^{-n} U_{j}^{1}\right)}\|h\|_{u} \\
& \quad \leq C\|h\|_{u} \lambda^{-n \beta} \varepsilon^{\beta}\left(D_{n}+\rho^{n}\right),
\end{aligned}
$$

where we have used Lemma 3.2 in the last line with $\varsigma=0$.

It remains to estimate the second sum in (4.11) using the strong stable norm. We need the following fact.

Lemma 4.1. For each $j$, we have that

$$
\begin{aligned}
& \left|\left(\left|D T^{n}\right|^{-1} J_{W^{1}} T^{n}\right) \circ G_{F_{j}^{1}}-\left(\left|D T^{n}\right|^{-1} J_{W^{2}} T^{n}\right) \circ G_{F_{j}^{2}}\right|_{\mathcal{C}^{q}} \\
& \quad \leq\left.\left. C|| D T^{n}\right|^{-1} J_{W^{1}} T^{n}\right|_{C^{0}\left(T^{-n} U_{j}^{1}\right)} \varepsilon^{1-q} .
\end{aligned}
$$

Proof. Throughout the proof, for ease of notation we write $J_{i}^{n}$ for $\left|D T^{n}\right|^{-1} J_{W^{i}} T^{n}$.

For any $t \in I_{r_{j}^{2}}, x=G_{F_{j}^{1}}(t)$ and $y=G_{F_{j}^{2}}(t)$ lie on a common element $\gamma \in \mathcal{F}^{u}$. Thus $T^{n}(x)$ and $T^{n}(y)$ also lie on the element $T^{n} \gamma \in \mathcal{F}^{u}$ which intersects $W^{1}$ and $W^{2}$ and has length at most $C \varepsilon$. By (A.1),

$$
\left|J_{1}^{n}(x)-J_{2}^{n}(y)\right| \leq C\left|J_{1}^{n}\right|_{\mathcal{C}^{0}\left(T^{-n} U_{j}^{1}\right)} d_{u}\left(T^{n} x, T^{n} y\right) \leq C \varepsilon\left|J_{1}^{n}\right|_{\mathcal{C}^{0}\left(T^{-n} U_{j}^{1}\right)} .
$$

Using this estimate and the fact that $J_{i}^{n} \circ G_{F_{j}^{i}}$ is $\mathcal{C}^{1}$, we write

$$
\begin{aligned}
& \frac{\left|\left(J_{1}^{n} \circ G_{F_{j}^{1}}(s)-J_{2}^{n} \circ G_{F_{j}^{2}}(s)\right)-\left(J_{1}^{n} \circ G_{F_{j}^{1}}(t)-J_{2}^{n} \circ G_{F_{j}^{2}}(t)\right)\right|}{|s-t|^{q}} \\
& \quad \leq \frac{2 C \varepsilon\left|J_{1}^{n}\right|_{\mathcal{C}^{0}\left(T^{-n} U_{j}^{1}\right)}}{|s-t|^{q}} .
\end{aligned}
$$

Also,

$$
\begin{aligned}
& \frac{\left|\left(J_{1}^{n} \circ G_{F_{j}^{1}}(s)-J_{1}^{n} \circ G_{F_{j}^{1}}(t)\right)-\left(J_{2}^{n} \circ G_{F_{j}^{2}}(s)-J_{2}^{n} \circ G_{F_{j}^{2}}(t)\right)\right|}{|s-t|^{q}} \\
& \quad \leq 2 C\left|J_{1}^{n}\right|_{\mathcal{C}^{0}\left(T^{-n} U_{j}^{1}\right)}|s-t|^{1-q} .
\end{aligned}
$$

Putting (4.16) and (4.17) together implies that the Hölder constant of $J_{1}^{n} \circ G_{F_{j}^{1}}-$ $J_{2}^{n} \circ G_{F_{j}^{2}}$ is bounded by

$$
H^{q}\left(J_{1}^{n} \circ G_{F_{j}^{1}}-J_{2}^{n} \circ G_{F_{j}^{2}}\right) \leq C\left|J_{1}^{n}\right|_{\mathcal{C}^{0}\left(T^{-n} U_{j}^{1}\right)} \min \left\{\varepsilon|s-t|^{-q},|s-t|^{1-q}\right\} .
$$


This expression is maximized when $\varepsilon|s-t|^{-q}=|s-t|^{1-q}$, i.e., when $\varepsilon=|s-t|$. Thus $H^{q}\left(J_{1}^{n} \circ G_{F_{j}^{1}}-J_{2}^{n} \circ G_{F_{j}^{2}}\right) \leq C\left|J_{1}^{n}\right|_{\mathcal{C}^{0}\left(T^{-n} U_{j}^{1}\right.} \varepsilon^{1-q}$, which, together with (4.15), concludes the proof of the lemma.

Using the strong stable norm, we estimate the second sum in (4.11) by

$$
\begin{aligned}
& \left.\sum_{j}\left|\int_{T^{-n} U_{j}^{2}} h\right| D T^{n}\right|^{-1} J_{W^{2}} T^{n}\left(\tilde{\varphi}-\varphi_{2}\right) \circ T^{n} \mid \\
& \quad \leq\left.\left. C\|h\|_{s} \sum_{j}\left|T^{-n} U_{j}^{2}\right|^{\alpha}|| D T^{n}\right|^{-1} J_{W^{2}} T^{n}\left(\tilde{\varphi}-\varphi_{2}\right) \circ T^{n}\right|_{\mathcal{C}^{q}\left(T^{-n} U_{j}^{2}\right)} .
\end{aligned}
$$

In order to estimate the $\mathcal{C}^{q}$-norm of the function in (4.18), we split it up into two differences,

$$
\begin{aligned}
& \|\left.\left. D T^{n}\right|^{-1} J_{W^{2}} T^{n} \cdot\left(\tilde{\varphi}-\varphi_{2}\right) \circ T^{n}\right|_{\mathcal{C}^{q}\left(T^{-n} U_{j}^{2}\right)} \\
\leq & C \mid\left[\left(\left|D T^{n}\right|^{-1} J_{W^{1}} T^{n}\right) \cdot \varphi_{1} \circ T^{n}\right] \circ G_{F_{j}^{1}} \\
& -\left.\left[\left(\left|D T^{n}\right|^{-1} J_{W^{2}} T^{n}\right) \cdot \varphi_{2} \circ T^{n}\right] \circ G_{F_{j}^{2}}\right|_{\mathcal{C}^{q}\left(I_{r_{j}}\right)} \\
\leq & C \mid\left(\left|D T^{n}\right|^{-1} J_{W^{1}} T^{n}\right) \circ G_{F_{j}^{1}}\left[\left.\left(\varphi_{1} \circ T^{n} \circ G_{F_{j}^{1}}-\varphi_{2} \circ T^{n} \circ G_{F_{j}^{2}}\right]\right|_{\mathcal{C}^{q}\left(I_{r_{j}}\right)}\right. \\
& +C\left|\left[\left(\left|D T^{n}\right|^{-1} J_{W^{1}} T^{n}\right) \circ G_{F_{j}^{1}}-\left(\left|D T^{n}\right|^{-1} J_{W^{2}} T^{n}\right) \circ G_{F_{j}^{2}}\right] \varphi_{2} \circ T^{n} \circ G_{F_{j}^{2}}\right|_{\mathcal{C}^{q}\left(I_{r_{j}}\right)} \\
\leq & \left.\left.C|| D T^{n}\right|^{-1} J_{W^{1}} T^{n}\right|_{\mathcal{C}^{0}\left(T^{-n} U_{j}^{1}\right)}\left|\varphi_{1} \circ T^{n} \circ G_{F_{j}^{1}}-\varphi_{2} \circ T^{n} \circ G_{F_{j}^{2}}\right|_{\mathcal{C}^{q}\left(I_{r_{j}}\right)} \\
& +C\left|\left(\left|D T^{n}\right|^{-1} J_{W^{1}} T^{n}\right) \circ G_{F_{j}^{1}}-\left(\left|D T^{n}\right|^{-1} J_{W^{2}} T^{n}\right) \circ G_{F_{j}^{2}}\right|_{\mathcal{C}^{q}\left(I_{r_{j}}\right)} \cdot
\end{aligned}
$$

Note that the second term can be bounded using Lemma 4.1 To bound the first term, let $F^{i} \in \Xi$ be the function defining $W^{i}$. Then setting $\alpha_{j}:=G_{F^{2}}^{-1} \circ T^{n} \circ G_{F_{j}^{2}}$, we have that $\left|\alpha_{j}\right|_{\mathcal{C}^{q}} \leq C$ and

$$
\begin{aligned}
&\left|\varphi_{1} \circ T^{n} \circ G_{F_{j}^{1}}-\varphi_{2} \circ T^{n} \circ G_{F_{j}^{2}}\right|_{\mathcal{C}^{q}\left(I_{r_{j}}\right)} \\
& \quad=\left|\varphi_{1} \circ T^{n} \circ G_{F_{j}^{1}} \circ \alpha_{j}^{-1} \circ \alpha_{j}-\varphi_{2} \circ T^{n} \circ G_{F_{j}^{2}} \circ \alpha_{j}^{-1} \circ \alpha_{j}\right|_{\mathcal{C}^{q}\left(I_{r_{j}}\right)} \\
&=\left|\varphi_{1} \circ \Psi \circ G_{F^{2}} \circ \alpha_{j}-\varphi_{2} \circ G_{F^{2}} \circ \alpha_{j}\right|_{\mathcal{C}^{q}\left(I_{r_{j}}\right)} \\
& \leq C\left|\varphi_{1} \circ \Psi \circ G_{F^{2}}-\varphi_{2} \circ G_{F^{2}}\right|_{\mathcal{C}^{q}\left(I_{r_{j}}\right)}+C\left|\varphi_{1} \circ G_{F^{1}}-\varphi_{2} \circ G_{F^{2}}\right|_{\mathcal{C}^{q}\left(I_{r_{j}}\right)} \\
& \leq C\left|\varphi_{1} \circ \Psi \circ G_{F^{2}}-\varphi_{1} \circ G_{F^{1}}\right|_{\mathcal{C}^{q}\left(I_{r_{j}}\right)}+\left.C{ }\right|_{\mathcal{C}^{q}\left(I_{r_{j}}\right)}+C d_{q}\left(\varphi_{1}, \varphi_{2}\right) . \\
& \leq C \mid \varphi_{1} \circ G_{F^{1}} \circ G_{F^{1}}^{-1} \odot \Psi \circ G_{F^{2}}-\varphi_{1} \circ G_{F^{1}}
\end{aligned}
$$

Thus we need the following final estimate.

Lemma 4.2. For a fixed $U_{j}^{2}$, let $J \subset I_{r_{2}}$ be an interval on which $G_{F^{1}}^{-1} \odot \Psi \circ G_{F^{2}}$ is defined. Then

$$
\left|I d-G_{F^{1}}^{-1} \circ \Psi \circ G_{F^{2}}\right|_{\mathcal{C}^{1}(J)} \leq C \varepsilon .
$$


Proof. Recall that $\Psi=T^{n} \circ G_{F_{j}^{1}} \circ G_{F_{j}^{2}}^{-1} \circ T^{-n}$. The function $\phi_{j}:=G_{F_{j}^{1}} \circ G_{F_{j}^{2}}^{-1}$ maps a point $x \in T^{-n} U_{j}^{2}$ to a point $y \in T^{-n} U_{j}^{1}$ which lies on an curve $\gamma \in \mathcal{F}^{u}$ containing both $x$ and $y$. Thus $\Psi$ maps $T^{n}(x)$ to $T^{n}(y)$ and these two points lie on $T^{n} \gamma \in \mathcal{F}^{u}$. By the transversality of the family $\mathcal{F}^{u}$, this implies that $d_{u}\left(T^{n} x, \Psi\left(T^{n} x\right)\right) \leq C \varepsilon$ where $d_{u}$ denotes distance along curves in $\mathcal{F}^{u}$. Then

$$
\begin{aligned}
\mid I d & -\left.G_{F^{1}}^{-1} \circ \Psi \circ G_{F^{2}}\right|_{\mathcal{C}^{0}(J)} \\
& =\left|G_{F^{1}}^{-1} \circ G_{F^{1}}-G_{F^{1}}^{-1} \circ \Psi \circ G_{F^{2}}\right| \leq\left|G_{F^{1}}^{-1}\right|_{\mathcal{C}^{1}}\left|G_{F^{1}}-\Psi \circ G_{F^{2}}\right| \\
& \leq(1+\kappa)\left(\left|G_{F^{1}}-G_{F^{2}}\right|+\left|G_{F^{2}}-\Psi \circ G_{F^{2}}\right|\right) \leq(1+\kappa)(\varepsilon+C \varepsilon) .
\end{aligned}
$$

Closeness in the $\mathcal{C}^{1}$-norm follows from the fact that all the functions involved are bounded in $\mathcal{C}^{2}$-norm, $\left|G_{F^{1}}-G_{F^{2}}\right|_{\mathcal{C}^{1}} \leq \varepsilon$, and

$$
|\partial \Psi-1|=\left|\partial\left(T^{n} \circ \phi_{j} \circ T^{-n}\right)-1\right|=\left|\frac{J_{W} T^{n}\left(\phi_{j} \circ T^{-n}\right)}{J_{W} T^{n}\left(T^{-n}\right)} \partial \phi_{j}-1\right| \leq C \varepsilon
$$

where $\partial$ denotes differentiation along $T^{-n} W^{2}$ and in the last inequality we have used distortion estimate (A.1).

We can now estimate equation (4.20) using Lemma 4.2

$$
\begin{aligned}
\left|\varphi_{1} \circ T^{n} \circ G_{F_{j}^{1}}-\varphi_{2} \circ T^{n} \circ G_{F_{j}^{2}}\right|_{\mathcal{C}^{q}\left(I_{r_{j}}\right)} \\
\quad \leq C\left|\varphi_{1} \circ G_{F^{1}} \circ\left(G_{F^{1}}^{-1} \circ \Psi \circ G_{F^{2}}-I d\right)\right|_{\mathcal{C}^{q}\left(I_{r_{j}}\right)}+C d_{q}\left(\varphi_{1}, \varphi_{2}\right) \\
\leq C\left|\varphi_{1} \circ G_{F^{1}}\right|_{\mathcal{C}^{q}\left(I_{r_{j}}\right)}\left|I d-G_{F^{1}}^{-1} \circ \Psi \circ G_{F^{2}}\right|_{\mathcal{C}^{1}\left(I_{r_{j}}\right)}+C \varepsilon \\
\leq C \varepsilon^{1-q}+C \varepsilon .
\end{aligned}
$$

The above, together with equation (4.19) and Lemma 4.1, implies (4.21)

$$
\left.\left.|| D T^{n}\right|^{-1} J_{W^{2}} T^{n} \cdot\left(\tilde{\varphi}-\varphi_{2}\right) \circ T^{n}\right|_{\mathcal{C}^{q}\left(T^{-n} U_{j}^{2}\right)} \leq\left.\left. C \varepsilon^{1-q}|| D T^{n}\right|^{-1} J_{W^{1}} T^{n}\right|_{\mathcal{C}^{0}\left(T^{-n} U_{j}^{2}\right)} .
$$

Since $1-q \geq \beta$, we can use (4.21) and (4.18) to estimate the second sum of (4.11) by

$$
\begin{aligned}
& \left.\sum_{j}\left|\int_{T^{-n} U_{j}^{2}} h\right| D T^{n}\right|^{-1} J_{W^{2}} T^{n}\left(\tilde{\varphi}-\varphi_{2}\right) \circ T^{n} \mid \\
& \quad \leq\left.\left. C\|h\|_{s} \varepsilon^{1-q} \sum_{j}\left|T^{-n} U_{j}^{2}\right|^{\alpha}|| D T^{n}\right|^{-1} J_{W^{2}} T^{n}\right|_{\mathcal{C}^{0}\left(T^{-n} U_{j}^{2}\right)} \\
& \quad \leq C\|h\|_{s} D_{n}\left|W^{2}\right|^{\alpha} \varepsilon^{\beta}
\end{aligned}
$$

where in the last line we have again used Lemma 3.2 with $\varsigma=\alpha$.

Combining the estimates from equations (4.10), (4.14), and (4.22), we obtain

$$
\left\|\mathcal{L}^{n} h\right\|_{u} \leq C\|h\|_{u} \lambda^{-\beta n} D_{n}+C\|h\|_{s}\left(D_{n}+L_{n} \lambda^{-n} \mu^{-\alpha n}\right) .
$$

This completes the proof of (2.9). 


\section{Spectral PiCture}

From the Lasota-Yorke estimates (2.10) and the compactness it follows by the standard Hennion argument (see [B1] for details) that the spectral radius of $\mathcal{L}$ is bounded by $\left(D_{N}\right)^{\frac{1}{N}}$ and the essential spectral radius by $\tau\left(D_{N}\right)^{\frac{1}{N}}$, where one can take $N$ arbitrary large provided $b$ is chosen sufficiently small. But since norms with different $b$ are all equivalent, the spectral radii are insensitive to the choice of $b$. Accordingly, fixing $b$ small enough once and for all, we see that the spectral radius of $\mathcal{L}$ is bounded by $D_{*}:=\limsup _{n \rightarrow \infty} D_{n}^{1 / n}$ and the essential spectral radius is bounded by $\tau D_{*}$. To proceed we need an estimate of $D_{*}$.

\subsection{Spectral radius.}

Lemma 5.1. Let $r$ be such that $\left\|\mathcal{L}^{n}\right\| \leq C r^{n}$. Then $D_{n} \leq \delta^{\alpha-1} C r^{n}$.

Proof. For each $W \in \Sigma$,

$$
\delta^{\alpha-1}|W|^{-\alpha} \int_{W}\left|D T^{-k}\right| d m=\delta^{\alpha-1}|W|^{-\alpha} \int_{W} \mathcal{L}^{k} 1 \leq \delta^{\alpha-1}\left\|\mathcal{L}^{k} 1\right\|_{s} \leq \delta^{\alpha-1} C r^{k}
$$

Taking the supremum over $W$ and $0 \leq k \leq n$ yields the lemma.

Remark 5.2. Lemma 5.1 implies that $D_{*}$ is bounded by the spectral radius of $\mathcal{L}$ and since the Lasota-Yorke estimates imply the reverse inequality, we conclude that in fact $D_{*}$ is the spectral radius of $\mathcal{L}$ on $\mathcal{B}$.

Thanks to Lemma 3.3 and Lemma 5.1 we can prove the following characterization.

Lemma 5.3. The spectral radius of $\mathcal{L}$ on $\mathcal{B}$ is one and the essential spectral radius is $\tau$. In addition, calling $\mathbb{V}$ the eigenspace associated to the eigenvalues of modulus one, then $\mathcal{L}$ restricted to $\mathbb{V}$ has a semi-simple spectrum (no Jordan blocks). Finally, $\mathbb{V}$ consists of signed measures.

Proof. Recall that by quasi-compactness, the part of the spectrum larger than $\tau D_{*}$ is of finite rank (see [B1]). Now, let $z$ be in the spectrum of $\mathcal{L},|z|>\max \left\{1, \tau D_{*}\right\}$. Then there must exist an $h \in \mathcal{B}$ such that $\mathcal{L} h=z h$. Accordingly, for each $\varphi \in \mathcal{C}^{1}$, since $\varphi \circ T^{n} \in \mathcal{C}_{S_{n}^{+}}^{1}$ for all $n \in \mathbb{N}$,

$$
|h(\varphi)|=|z|^{-n}\left|\mathcal{L}^{n} h(\varphi)\right| \leq|z|^{-n}\left|h\left(\varphi \circ T^{n}\right)\right| \leq|z|^{-n} C\|h\|\left(|\varphi|_{\infty}+\mu_{+}^{n}\left|D^{s} \varphi\right|_{\infty}\right)
$$

by Lemma 3.3 .

Thus, if $|z|>1$, we have $h(\varphi)=0$ for each $\varphi \in \mathcal{C}^{1}$, which implies $h=0$ by Remark 3.4. On the other hand, if $|z|=1$, then it follows that $|h(\varphi)| \leq C\|h\| \cdot|\varphi|_{\infty}$, so $h$ is a measure.

Next, suppose $\tau D_{*} \geq 1$. By the preceding paragraph, the spectral radius of $\mathcal{L}$ can be at most $\tau D_{*}$, thus $D_{*} \leq \tau D_{*}$, which is impossible since $\tau<1$. Hence, the spectral radius is $D_{*}=1$.

It remains to show that there are no Jordan blocks corresponding to the peripheral spectrum. Indeed, suppose that there exists $z \in \mathbb{C}$ and $h_{0}, h_{1} \in \mathcal{B}$ such that $|z|=1$ and $h_{0} \neq 0, \mathcal{L} h_{0}=z h_{0}, \mathcal{L} h_{1}=z h_{1}+h_{0}$. This would imply $z^{-n} \mathcal{L}^{n} h_{1}=n z^{-1} h_{0}+h_{1}$, and thus

$$
n\left|h_{0}(\varphi)\right| \leq\left|h_{1}(\varphi)\right|+C\left\|h_{1}\right\|\left(|\varphi|_{\infty}+\mu_{+}^{n}\left|D^{s} \varphi\right|_{\infty}\right)
$$


Dividing by $n$ and taking the limit as $n$ approaches infinity, it follows that $h_{0}=0$, contrary to the hypothesis.

Remark 5.4. Note that Lemma 5.3 implies $\left\|\mathcal{L}^{n}\right\| \leq C$ for each $n \in \mathbb{N}$, hence Lemma 5.1 implies $D_{n} \leq C_{\delta}$ for all $n \in \mathbb{N}$.

5.2. Peripheral spectrum. The following two lemmas prove Theorem 2.8, points (1)-(4) and part of (5). The rest is proved in Section 5.3.

Let $\mathbb{V}_{\theta}$ be the eigenspace associated to the eigenvalue $e^{2 \pi i \theta}$. For the rest of this section, we use $m$ to denote normalized Riemannian volume (Lebesgue measure) on $\mathcal{M}$.

Lemma 5.5. Recall that $\bar{\mu}:=\Pi_{0} 1$. Then,

(i) All the measures in $\mathbb{V}$ are absolutely continuous with respect to $\bar{\mu}$. Moreover, 1 belongs to the spectrum.

(ii) There exists a finite number of $q_{i} \in \mathbb{N}$ such that the spectrum on the unit disk is $\bigcup_{k}\left\{e^{2 \pi i \frac{p}{q_{k}}}: 0<p \leq q_{k}, p \in \mathbb{N}\right\}$. In addition, the set of ergodic probability measures absolutely continuous with respect to $\bar{\mu}$ form a basis of $\mathbb{V}_{0}$.

(iii) For each $\mu \in \mathbb{V}, n \in \mathbb{N}$, we have $\mu\left(\mathcal{S}_{n, \epsilon}^{ \pm}\right) \leq C_{n} \epsilon^{\alpha}$. In particular, $\mu\left(\mathcal{S}_{n}^{ \pm}\right)=0$.

Proof. (i) Let $\Pi_{\theta}$ be the eigenprojector on $\mathbb{V}_{\theta}$. The fact that the spectrum outside the circle of radius $\tau$ consists of only finitely many eigenvalues of finite multiplicity implies that the limit

$$
\lim _{n \rightarrow \infty} \frac{1}{n} \sum_{k=0}^{n-1} e^{-2 \pi i \theta k} \mathcal{L}^{k}=\Pi_{\theta}
$$

is well-defined in the uniform topology of $L(\mathcal{B}, \mathcal{B})$. Moreover, $\Pi_{0}$ is obviously a positive operator and, by density, $\mathbb{V}_{\theta}=\Pi_{\theta} \mathcal{C}^{1}$.

Accordingly, for each $\mu \in \mathbb{V}_{\theta}$, there exists $h \in \mathcal{C}^{1}$ such that $\Pi_{\theta} h=\mu$. Thus, for each $\varphi \in \mathcal{C}^{1}$

$$
|\mu(\varphi)|=\left|\Pi_{\theta} h(\varphi)\right| \leq|h|_{\infty} \Pi_{0} 1(|\varphi|)=:|h|_{\infty} \bar{\mu}(|\varphi|) .
$$

That is, each probability measure $\mu \in \mathbb{V}_{\theta}$ is absolutely continuous with respect to $\bar{\mu}$. Moreover, setting $h_{\mu}:=\frac{d \mu}{d \bar{\mu}}$, we have $h_{\mu} \in L^{\infty}(\mathcal{M}, \bar{\mu})$. This implies $\bar{\mu} \neq 0$; otherwise the spectral radius of $\mathcal{L}$ would be strictly smaller than one, which, recalling Remark 3.4, yields the contradiction

$$
1=|m(1)|=\left|\mathcal{L}^{n} m(1)\right|=\lim _{n \rightarrow \infty}\left|\mathcal{L}^{n} m(1)\right| \leq \lim _{n \rightarrow \infty} C\left\|\mathcal{L}^{n} m\right\|=0 .
$$

Hence one belongs to the spectrum.

(ii) $\quad$ Next, for $\mu \in \mathbb{V}_{\theta}$ and each $\varphi \in \mathcal{C}^{1}$,

$$
\begin{aligned}
\int \varphi h_{\mu} d \bar{\mu} & =\mu(\varphi)=e^{-2 \pi i \theta} \mathcal{L} \mu(\varphi)=e^{-2 \pi i \theta} \mu(\varphi \circ T) \\
& =e^{-2 \pi i \theta} \int \varphi \circ T h_{\mu} d \bar{\mu}=e^{-2 \pi i \theta} \int \varphi h_{\mu} \circ T^{-1} d \bar{\mu} .
\end{aligned}
$$


Accordingly $h_{\mu} \circ T^{-1}=e^{2 \pi i \theta} h_{\mu}, \bar{\mu}$ a.e. This in turn means that, setting, $h_{\mu, k}:=$ $\left(h_{\mu}\right)^{k} \in L^{\infty}(\mathcal{M}, \bar{\mu})$, since the measure $d \mu_{k}:=h_{\mu, k} d \bar{\mu}$ belongs to $\mathcal{B}$ for each $k \in \mathbb{N} 9$ then $\mathcal{L} \mu_{k}=e^{2 \pi i k \theta} \mu_{k}$. That is, $e^{2 \pi i k \theta}$ belongs to the peripheral spectrum and since the peripheral spectrum consists of a finite number of points, it must be that $\theta \in \mathbb{Q}$.

Now let $\mu \in \mathbb{V}_{0}$ and choose $h \in \mathcal{C}^{1}$ such that $\mu=\Pi_{0} h$. We can then write $h=h_{+}-h_{-}, h_{ \pm}:=\max \{0, \pm h\}$. Since $h_{ \pm}$are Lipschitz functions, they belong to $\mathcal{B}$. We can then define $\mu_{ \pm}:=\Pi_{1} h_{ \pm}$. Thus $\mathbb{V}_{0}$ is the span of a convex set of probability measures.

Next, assume that $\Omega$ is an invariant set of positive $\bar{\mu}$ measure; then for each $\varepsilon>0$ there exists a smooth function $\varphi_{\varepsilon}>0$ such that $\bar{\mu}\left(\left|\mathbf{I d}_{\Omega}-\varphi_{\varepsilon}\right|\right) \leq \varepsilon$. Thus, for each continuous function $\phi$

$\bar{\mu}\left(\varphi_{\varepsilon} \phi \circ T^{n}\right)=\bar{\mu}\left(\mathbf{I d}_{\Omega} \phi \circ T^{n}\right)+\mathcal{O}\left(\varepsilon|\phi|_{\infty}\right)=\bar{\mu}\left(\mathbf{I d}_{\Omega} \phi\right)+\mathcal{O}\left(\varepsilon|\phi|_{\infty}\right)=\bar{\mu}\left(\varphi_{\varepsilon} \phi\right)+\mathcal{O}\left(\varepsilon|\phi|_{\infty}\right)$.

On the other hand

$$
\lim _{n \rightarrow \infty} \frac{1}{n} \sum_{k=0}^{n-1} \bar{\mu}\left(\varphi_{\varepsilon} \phi \circ T^{n}\right)=\Pi_{1}\left(\bar{\mu}_{\varepsilon}\right)(\phi)
$$

where $\bar{\mu}_{\varepsilon}(\phi):=\bar{\mu}\left(\varphi_{\varepsilon} \phi\right)$. By the arbitrariness of $\varepsilon$ it follows, setting $\bar{\mu}_{\Omega}(\phi):=$ $\bar{\mu}\left(\mathbf{I d}_{\Omega} \phi\right)$, that $\bar{\mu}_{\Omega} \in \mathbb{V}_{0}$ and, since $\mathbb{V}_{0}$ is finite dimensional, that there are only finitely many invariant sets of positive $\bar{\mu}$ measure and the ergodic decomposition yields a basis of $\mathbb{V}_{0}$.

(iii) $\quad$ Finally, let $\mu \in \mathbb{V}$. By hypothesis, the tangent space of $\mathcal{S}_{m}^{-}$is bounded away from $C^{s}$. Calling $\mathcal{S}_{m, \epsilon}^{-}$an $\epsilon$ neighborhood of $\mathcal{S}_{m}^{-}$, set $\mu_{\epsilon}(\varphi):=\mu\left(\mathbf{I d}_{\mathcal{S}_{m, \epsilon}^{-}} \varphi\right)$. Let $h_{n}$ be a sequence that converges to $\mu$ in $\mathcal{B}$; then it is immediate to check that $h_{n, \epsilon}(\varphi):=h_{n}\left(\mathbf{I d}_{\mathcal{S}_{m, \epsilon}^{-}} \varphi\right)$ belongs to $\mathcal{B}_{w}$. In addition,

$$
\int_{W} \varphi h_{n, \epsilon} d m=\int_{W \cap \mathcal{S}_{m, \epsilon}^{-}} \varphi h_{n} d m \leq C_{m}\left\|h_{n}\right\| \epsilon^{\alpha},
$$

for $\varphi \in \mathcal{C}^{1}(W)$. In the same way one has that $h_{n, \epsilon}$ is a Cauchy sequence in $\mathcal{B}_{w}$, thus it must converge to $\mu_{\epsilon}(\varphi):=\mu\left(\operatorname{Id}_{\mathcal{S}_{m, \epsilon}^{-}} \varphi\right)$. Since $\mu_{\epsilon}(1) \leq C_{m} \epsilon^{\alpha}$, the regularity of $\mu$ implies $\mu\left(\mathcal{S}_{m}^{-}\right)=0$. The result follows since $T \mathcal{S}^{+}=\mathcal{S}^{-}$.

Remark 5.6. For uniformly hyperbolic maps with singularities, the estimate $\bar{\mu}\left(\mathcal{S}_{\epsilon}^{ \pm}\right)$ $\leq C \epsilon^{\alpha}$ is enough to conclude the existence of stable and unstable manifolds for $\bar{\mu}$-a.e. $x \in \mathcal{M}$ using the standard Borel-Cantelli argument (see, for example, [LW]). In fact, from the ergodic decomposition proved in Lemma 5.7, it follows that stable and unstable manifolds exist for $m$-a.e. $x \in \mathcal{M}$.

Recall that to each physical measure $\mu$ is associated a positive Lebesgue measure invariant set $B_{\mu}$ such that, for every continuous function $f$,

$$
\lim _{n \rightarrow \infty} \frac{1}{n} \sum_{i=0}^{n-1} f\left(T^{i} x\right)=\mu(f) \quad \forall x \in B_{\mu} .
$$

${ }^{9}$ Just consider $h \in \mathcal{C}^{1}$ such that $\bar{\mu}\left(\left|h-h_{\mu, k}\right|\right) \leq \varepsilon$. Then setting $d \nu:=h d \bar{\mu}$,

$$
\left(\Pi_{k \theta} \nu-\mu_{k}\right)(\varphi) \leq \lim _{n \rightarrow \infty} \frac{1}{n} \sum_{j=0}^{n-1} \bar{\mu}\left(\left|h-h_{\mu, k}\right| \circ T^{-j}\right)\left|\varphi_{\infty}\right| \leq \varepsilon|\varphi|_{\infty} .
$$

Hence, $\mu_{k}$ is an accumulation point of elements of $\mathbb{V}$ and so it belongs to $\mathbb{V}$. 
Lemma 5.7. Let $T$ be a piecewise uniformly hyperbolic map as described in Section 2 .

(i) $T$ admits only finitely many physical probability measures, and they belong to $\mathbb{V}_{0}$.

(ii) The ergodic decomposition with respect to Lebesgue and with respect to $\bar{\mu}$ coincide.

(iii) The forward average for each continuous function is well defined m-almost everywhere and the ergodic decomposition with respect to Lebesgue corresponds to the supports of the physical measures.

Proof. (i) Let $\mu$ be a physical measure and take a density point $x$ of the associated set $B_{\mu}$. Then for each $\varepsilon>0$ there exists an open set $U$ containing $x$ such that $m\left(B_{\mu} \cap U\right) \geq(1-\varepsilon) m(U)$. Consider a smooth probability measure $\mu_{U}$ supported in $U$, such that $\mu_{U}\left(B_{\mu}\right) \geq 1-2 \varepsilon$. Then for each $f \in \mathcal{C}^{0}$,

$$
\begin{aligned}
\Pi_{1} \mu_{U}(f)= & \lim _{n \rightarrow \infty} \frac{1}{n} \sum_{k=0}^{n-1} \mu_{U}\left(f \circ T^{i}\right)=\lim _{n \rightarrow \infty} \frac{1}{n} \sum_{k=0}^{n-1} \mu_{U}\left(f \circ T^{i} \mathbf{I d}_{B_{\mu}}\right)+\mathcal{O}\left(|f|_{\infty} \varepsilon\right) \\
& =\mu_{U}\left(\mathbf{I d}_{B_{\mu}}\right) \mu(f)+\mathcal{O}\left(|f|_{\infty} \varepsilon\right)=\mu(f)+\mathcal{O}\left(|f|_{\infty} \varepsilon\right) .
\end{aligned}
$$

This means that $\mu$ can be approximated by elements of $\mathbb{V}_{0}$ and therefore $\mu \in \mathbb{V}_{0}$. In addition, since the physical measures are ergodic by definition, it follows that they must belong to the ergodic elements of $\mathbb{V}_{0}$.

(ii) Consider an invariant set $A$ of positive $\bar{\mu}$ measure such that $\bar{\mu}$ restricted to $A$ is ergodic. If we consider the set $A_{u}:=\bigcup_{x \in A} W^{u}(x) 10$ then $T^{-1} A_{u} \subset A_{u}$ and $\bar{\mu}\left(A_{u} \backslash A\right)=0$. Indeed, for each continuous function $\varphi$ the backward average on $A_{u}$ coincides with the backward average on $A$ and hence equals $\bar{\mu}\left(\varphi \mathbf{I} \mathbf{d}_{A}\right) \bar{\mu}(A)^{-1}$ $\bar{\mu}$-a.e., by ergodicity. In addition, for each $\varepsilon>0$ there exists $\varphi \in \mathcal{C}^{0}$ such that $\bar{\mu}\left(\left|\varphi-\mathbf{I d}_{A}\right|\right) \leq \varepsilon$. But then

$$
\lim _{n \rightarrow \infty} \frac{1}{n} \sum_{k=0}^{n-1} \varphi \circ T^{-k} \mathbf{I d}_{A_{u}}=\bar{\mu}\left(\varphi \mathbf{I d}_{A}\right) \bar{\mu}(A)^{-1} \mathbf{I d}_{A_{u}} \quad \bar{\mu} \text {-a.e. }
$$

Integrating, we have $\bar{\mu}\left(\varphi \mathbf{I d}_{A_{u}}\right)=\bar{\mu}\left(\varphi \mathbf{I} \mathbf{d}_{A}\right) \bar{\mu}(A)^{-1} \bar{\mu}\left(A_{u}\right)$, which yields

$$
\bar{\mu}(A)=\bar{\mu}\left(A_{u}\right)+\mathcal{O}(\varepsilon)
$$

which, by the arbitrariness of $\varepsilon$, proves the claim.

Next, consider the neighborhood of the singularity $\mathcal{S}_{\delta}$. By Lemma 5.5(iii) it follows that $\bar{\mu}\left(\mathcal{S}_{\delta}\right) \leq C \delta^{\alpha}$; hence setting $\mathcal{S}_{\delta}^{*}:=\bigcup_{m \in \mathbb{Z}} T^{m} \mathcal{S}_{\delta|m|^{-2 / \alpha}}$ we have $\bar{\mu}\left(\mathcal{S}_{\delta}^{*}\right) \leq$ $C \delta^{\alpha} \sum_{m \in \mathbb{Z}}|m|^{-2} \leq C \delta^{\alpha}$. Hence, by choosing $\delta$ small enough and setting $B:=$ $A_{u} \backslash \mathcal{S}_{\delta}^{*}$, it holds that $\bar{\mu}(B)>0$. In addition, for each $x \in B$ the manifolds $W^{u}(x), W^{s}(x)$ have length at least $\delta$ (see, e.g., [LW]). Define $B_{\epsilon}$ to be the $\epsilon$ neighborhood of $B$.

Finally, for each $n \in \mathbb{N}$ let $U_{n}=\bigcup_{\substack{K \in \mathcal{K}_{n} \\ K \cap B \neq \emptyset}} K \bigcap B_{\delta / 2}$, and $\tilde{U}_{n}:=U_{n} \bigcap B_{\delta / 4}$; clearly $U_{n+1} \subseteq U_{n}$. Setting $\tilde{B}:=\bigcap_{n \in \mathbb{N}} U_{n}$, we have $\bar{\mu}\left(\tilde{B} \backslash A_{u}\right)=0$. Indeed, if $x \in \tilde{B} \backslash A_{u}$, then, for each $n \in \mathbb{N}$, there exist $y_{n} \in A_{u} \backslash \mathcal{S}_{\delta}^{*}$ and $K_{n} \in \mathcal{K}_{n}$ such that $x, y_{n} \in K_{n}$. On the other hand each $W^{s}\left(y_{n}\right)$ has size $\delta$, by construction.

\footnotetext{
${ }^{10} \mathrm{By} W^{u}(x), W^{s}(x)$ we designate the unstable and stable manifolds of $x$, which exists $\bar{\mu}$-a.e. (see Remark 5.6). Hence, by eventually changing $A$ on a set of zero $\bar{\mu}$-measure, we can assume $A \subset A_{u}$.
} 
We can then extract a converging subsequence $\left\{y_{n_{j}}\right\}$, and call $W$ the limit of $W^{s}\left(y_{n}\right)$. It is easy to check that $W$ is a stable manifold, and since in the transversal direction the map is expanding, it must be $W=\bigcap_{n} K_{n}$. Accordingly, $W \subset W^{s}(x)$. The uniform transversality of the stable and unstable manifolds then implies that $W^{u}\left(y_{n}\right) \cap W^{s}(x) \neq \emptyset$ for $n$ large enough. Hence, $W^{s}(x) \cap A_{u} \neq \emptyset$, that is, $\tilde{B} \subset$ $A_{u, s}:=\bigcup_{x \in A_{u}} W^{s}(x)$. The claim then follows since one can prove $\bar{\mu}\left(A_{u, s} \backslash A_{u}\right)=0$ by the same argument that established $\bar{\mu}\left(A_{u} \backslash A\right)=0$.

In addition, by the regularity of both $\bar{\mu}$ and $m$, for each $k \in \mathbb{N}$ and $\varepsilon>0$, there exists $n_{\varepsilon}$ such that

$$
\bar{\mu}\left(U_{n_{\varepsilon}} \backslash \tilde{B}\right)+\sum_{j=0}^{k} \mathcal{L}^{k} m\left(U_{n_{\varepsilon}} \backslash \tilde{B}\right) \leq \varepsilon .
$$

Let $\phi$ be a smooth function such that $\phi_{\Gamma_{B_{\delta / 4}}}=1$ and $\operatorname{supp} \phi$ is contained in $B_{\delta / 2}$. Clearly $\phi \mathbf{I d}_{U_{n}} \in \mathcal{C}_{\mathcal{S}_{n}^{+}}^{1}$; we can then use the above inequality and Lemma 3.3 to write

$$
\begin{aligned}
& \bar{\mu}(\tilde{B})=\bar{\mu}\left(\tilde{U}_{n_{\varepsilon}}\right)+\mathcal{O}(\varepsilon) \leq \bar{\mu}\left(\mathbf{I d}_{U_{n_{\varepsilon}}} \phi\right)+\mathcal{O}(\varepsilon)=\frac{1}{k} \sum_{j=0}^{l-1} \mathcal{L}^{j} m\left(\mathbf{I d}_{U_{n_{\varepsilon}}} \phi\right)+\mathcal{O}\left(k^{-1}+\varepsilon\right) \\
& =\frac{1}{k} \sum_{j=0}^{l-1} \mathcal{L}^{j} m(\tilde{B})+\mathcal{O}\left(k^{-1}+\varepsilon\right) \leq \frac{1}{k} \sum_{j=0}^{l-1} \mathcal{L}^{j} m\left(A_{u, s}\right)+\mathcal{O}\left(k^{-1}+\varepsilon\right) \\
& =m\left(A_{u, s}\right)+\mathcal{O}\left(k^{-1}+\varepsilon\right) .
\end{aligned}
$$

By first choosing $k$ large enough and then $\varepsilon$ sufficiently small, we have $m\left(A_{u, s}\right)>0$.

Next notice that if $A_{u, s}$ is not an ergodic component for $m$, then there exists an invariant set of positive $m$-measure that will support a physical measure. But such a measure would belong to $\mathbb{V}_{0}$ by point (i), and this would be a contradiction.

(iii) The preceding argument also implies that if $\mu_{i}$ is a basis of $\mathbb{V}_{0}$ made of ergodic measures, then they are physical measures and $\left\{B_{\mu_{i}}\right\}$ corresponds to the ergodic decomposition with respect to Lebesgue. In addition, since $m\left(B_{\mu_{i}}\right) \geq$ $\bar{\mu}\left(B_{\mu_{i}}\right)$ and $\sum_{i} \bar{\mu}\left(B_{\mu_{i}}\right)=1$, the inequality must be an equality, and the forward average for each continuous function is well defined $m$-almost everywhere.

5.3. Statistical properties and Ruelle resonances. In addition to providing information about the invariant measures, the established spectral picture has other far reaching implications. To discuss them let us define the correlation functions. For each $f, g \in \mathcal{C}^{\beta}$ define

$$
C_{f, g}(n):=\bar{\mu}\left(f g \circ T^{n}\right)-\bar{\mu}(f) \bar{\mu}(g) .
$$

If the system is mixing (that is, one is the only eigenvalue on the unit circle and it is simple), then for each $\sigma$ larger than the norm of the second largest eigenvalue (or $\tau$ if no other eigenvalue is present outside the essential spectral radius) there holds

$$
\left|C_{f, g}(n)\right| \leq C \sigma^{n}|f|_{\mathcal{C}^{\beta}}|g|_{\mathcal{C}^{\beta}} .
$$

In other words we have the well-known dichotomy: either the system does not mix or it mixes exponentially fast (on Hölder observables).

More generally, we can define the Fourier transform of the correlation function:

$$
\hat{C}_{f, g}(z):=\sum_{n \in \mathbb{Z}} z^{n} C_{f, g}(n) .
$$


The above quantity is widely used in physics literature where usually one assumes that it is convergent in a neighborhood of $|z|=1$ (here this already follows from (5.3) ) and it has a meromorphic extension on some larger annulus. The poles of such a quantity are, in principle, measurable in a physical system and are called Ruelle resonances (see [Ru1, Ru2, PP1, PP2]). Due to our results we can substantiate the above picture for the class of systems at hand.

Indeed, note that we can assume, without loss of generality, $\bar{\mu}(f)=\bar{\mu}(g)=0$ and that if we define $\mu_{f}(\varphi):=\bar{\mu}(f \varphi), \mu_{g}(\varphi):=\bar{\mu}(g \varphi)$, then $\mu_{f}, \mu_{g} \in \mathcal{B}$. Thus,

$$
\begin{aligned}
\hat{C}_{f, g}(z) & =\sum_{n=0}^{\infty} z^{n} \bar{\mu}\left(f g \circ T^{n}\right)+\sum_{n=0}^{\infty} z^{-n} \bar{\mu}\left(f \circ T^{n} g\right)-\bar{\mu}(f g) \\
& =\sum_{n=0}^{\infty} z^{n} \mathcal{L}^{n} \mu_{f}(g)+\sum_{n=0}^{\infty} z^{-n} \mathcal{L}^{n} \mu_{g}(f)-\bar{\mu}(f g) \\
& =(z-\mathcal{L})^{-1} \mu_{f}(g)+\left(z^{-1}-\mathcal{L}\right)^{-1} \mu_{g}(f)-\bar{\mu}(f g) .
\end{aligned}
$$

It is thus obvious that the desired meromorphic extension is provided by the resolvent and that the poles are in one-to-one correspondence (including multiplicity) with the spectrum of $\mathcal{L}$. More precisely we have a meromorphic extension in the annulus $\left\{z \in \mathbb{C}: \tau<|z|<\tau^{-1}\right\}$.

Remark 5.8. Note that the above fact shows that the spectral data of the operator $\mathcal{L}$ on $\mathcal{B}$ is not a mathematical artifact but has a well-defined meaning which does not depend on any of the many arbitrary choices we have made in the construction of our functional analytic setting.

Remark 5.9. In the present situation the best one can do is to choose $\alpha=\beta=q=\frac{1}{2}$; moreover, if one assumes that $M(n)$ grows sub-exponentially (this is the case for billiards), then one has (assuming for simplicity $\lambda^{-1}=\mu_{+}$) that $\tau$ can be chosen arbitrarily close to $\lambda^{-\frac{1}{2}}$. At the moment it is unclear if such an estimate for the size of the meromorphic extension is real or is an artifact of the method of proof.

Another result that can be easily obtained by the present method is the Central Limit Theorem. Let $f \in \mathcal{C}^{\beta}$ with $\bar{\mu}(f)=0$ and define $S_{n}(f):=\sum_{k=0}^{n-1} f \circ T^{k}$. Then

$$
\bar{\mu}\left(e^{-i z S_{n}}\right)=\mathcal{L}_{z}^{n} \bar{\mu}(1)
$$

where $\mathcal{L}_{z}$ is the operator defined by $\mathcal{L}_{z} h(\varphi):=h\left(e^{-i z f} \varphi \circ T\right)$. Since $\mathcal{L}_{z}$ depends analytically on $z$, one can use standard perturbation theory to show that the leading eigenvalue is given by $1-\sigma z^{2}$, where $\sigma$ is the variance. Accordingly

$$
\lim _{n \rightarrow \infty} \bar{\mu}\left(e^{-i \frac{z}{\sqrt{n}} S_{n}}\right)=\lim _{n \rightarrow \infty}\left(1-\frac{\sigma z^{2}}{n}\right)^{n}=e^{-\sigma z^{2}}
$$

which is exactly the CLT. Other types of results (e.g. large deviations) can be approached along similar lines (see [HH, $\mathrm{CG}$ ] for more details).

\section{Perturbation Results}

Recall from Section 2.4 the set $\Gamma_{B_{*}}$ of maps $\tilde{T}$ that satisfy the same assumptions as $T$ in Section 2. In this section we derive results for several classes of perturbations and prove Theorems 2.13 and 2.18 . 


\subsection{Deterministic perturbations.}

Lemma 6.1. If two maps $T_{1}, T_{2} \in \Gamma_{B_{*}}$ satisfy $\gamma\left(T_{1}, T_{2}\right) \leq \varepsilon \leq \varepsilon_{0}$, then for each $h \in \mathcal{B}$,

$$
\left|\mathcal{L}_{T_{1}} h-\mathcal{L}_{T_{2}} h\right|_{w} \leq C_{b} \varepsilon^{\beta}\|h\|
$$

Proof. For $\varepsilon \leq \varepsilon_{0}$, we may choose the set of approximate stable leaves $\Sigma$ so that $T_{i}^{-1} \Sigma \subset \Sigma$ for $i=1,2$, and similarly for the approximate unstable family $\mathcal{F}^{u}$.

We first fix a leaf $W \in \Sigma$ and $\varphi$ with $|\varphi|_{\mathcal{C}^{1}(W)} \leq 1$ and write

$$
\int_{W}\left(\mathcal{L}_{T_{1}}-\mathcal{L}_{T_{2}}\right) h \varphi d m=\int_{T_{1}^{-1} W} h\left|D T_{1}\right|^{-1} J_{W} T_{1} \varphi \circ T_{1}-\int_{T_{2}^{-1} W} h\left|D T_{2}\right|^{-1} J_{W} T_{2} \varphi \circ T_{2} .
$$

Away from singularities, $T_{1}^{-1} W$ and $T_{2}^{-1} W$ are $\varepsilon$-close so we may partition $T_{1}^{-1} W$ and $T_{2}^{-1} W$ as we did in Section 4.3 .

Let $N_{\varepsilon}^{-}$denote the $\varepsilon$ neighborhood of the union of the singularity curves of $T_{1}^{-1}$ and $T_{2}^{-1}$. Consider one component $U_{j}$ of $W \backslash N_{\varepsilon}^{-}$. By assumption, we may choose functions $F_{j}^{i}$ defining the curves $T_{i}^{-1} U_{j}$ such that $d_{\Sigma}\left(T_{1}^{-1} U_{j}, T_{2}^{-1} U_{j}\right) \leq \varepsilon$. (If $\max \left\{\left|T_{1}^{-1} U_{j}\right|,\left|T_{2}^{-1} U_{j}\right|\right\}>2 \delta$, we further subdivide $U_{j}$ so that all components of $T_{1}^{-1} U_{j}$ and $T_{2}^{-1} U_{j}$ have length between $\delta$ and $2 \delta$.)

Denote by $V_{j}$ the connected components of $W \cap N_{\varepsilon}^{-}$and note that $\left|V_{j}\right| \leq C \varepsilon$ and that there are at most $L+2$ such pieces.

We estimate the integrals over the pieces $T_{i}^{-1} V_{j}$ similar to (4.10):

$$
\sum_{i, j} \int_{T_{i}^{-1} V_{j}} h\left|D T_{i}\right|^{-1} J_{W} T_{i} \varphi \circ T_{i} d m \leq C\|h\|_{s} \sum_{i, j}\left|V_{j}\right|^{\alpha} \lambda^{-1} \mu^{-\alpha} \leq C\|h\|_{s} \varepsilon^{\alpha}
$$

We split up the integrals over the $T_{i}^{-1} U_{j}$ as follows:

$$
\begin{aligned}
\sum_{j} & \int_{T_{1}^{-1} U_{j}} h\left|D T_{1}\right|^{-1} J_{W} T_{1} \varphi \circ T_{1} d m-\int_{T_{2}^{-1} U_{j}} h\left|D T_{2}\right|^{-1} J_{W} T_{2} \varphi \circ T_{2} d m \\
= & \sum_{j} \int_{T_{1}^{-1} U_{j}} h\left|D T_{1}\right|^{-1} J_{W} T_{1} \varphi \circ T_{1} d m-\int_{T_{2}^{-1} U_{j}} h f d m \\
& +\sum_{j} \int_{T_{2}^{-1} U_{j}} h\left(f-\left|D T_{2}\right|^{-1} J_{W} T_{2} \varphi \circ T_{2}\right) d m
\end{aligned}
$$

where $f=\left[\left|D T_{1}\right|^{-1} J_{W} T_{1} \varphi \circ T_{1}\right] \circ G_{F_{j}^{1}} \circ G_{F_{j}^{2}}^{-1}$. Note that $d_{q}\left(\left|D T_{1}\right|^{-1} J_{W} T_{1} \varphi \circ T_{1}, f\right)=$ 0 so that the first term of (6.2) can be estimated by

$$
\left.\sum_{j}\left|\int_{T_{1}^{-1} U_{j}} h\right| D T_{1}\right|^{-1} J_{W} T_{1} \varphi \circ T_{1}-\int_{T_{2}^{-1} U_{j}} h f \mid \leq C \varepsilon^{\beta}\|h\|_{u} .
$$

We estimate the second term of (6.2) using the strong stable norm. We follow (4.19) to estimate the $\mathcal{C}^{q}$-norm of the functions involved:

$$
\begin{aligned}
& \left.\left.|f-| D T_{2}\right|^{-1} J_{W} T_{2} \varphi \circ T_{2}\right|_{\mathcal{C}^{q}\left(T_{2}^{-1} U_{j}\right)} \\
& \leq C\left|\left[\left|D T_{1}\right|^{-1} J_{W} T_{1} \varphi \circ T_{1}\right] \circ G_{F_{j}^{1}}-\left[\left|D T_{2}\right|^{-1} J_{W} T_{2} \varphi \circ T_{2}\right] \circ G_{F_{2}^{2}}\right|_{\mathcal{C}^{q}\left(I_{r_{j}}\right)} \\
& \leq C\left|\varphi \circ T_{1} \circ G_{F_{j}^{1}}-\varphi \circ T_{2} \circ G_{F_{2}^{2}}\right|_{\mathcal{C}^{q}\left(I_{r_{j}}\right)} \\
& \left.\quad+C\left|\left(\left|D T_{1}\right|^{-1} J_{W} T_{1}\right) \circ G_{F_{j}^{1}}-\left(\left|D T_{2}\right|^{-1} J_{W} T_{2}\right) \circ G_{F_{2}^{2}}\right|_{\mathcal{C}^{q}\left(I_{r_{j}}\right.}\right) .
\end{aligned}
$$


The first term can be bounded using an estimate analogous to (4.20) and Lemma 4.2. The second term can be bounded using Lemma 4.1 and the fact that $\left|T_{1}-T_{2}\right|_{\mathcal{C}^{2}}<\varepsilon$ on $U_{j}^{i}$. Putting these estimates together, we conclude that $\left.|f-| D T_{2}\right|^{-1} J_{W} T_{2} \varphi$ 。 $\left.T_{2}\right|_{C^{q}} \leq C \varepsilon^{1-q}$ so we may estimate the second term of 6.2) by

$$
\int_{T_{2}^{-1} U_{j}} h\left(f-\left|D T_{2}\right|^{-1} J_{W} T_{2} \varphi \circ T_{2}\right) \leq C \varepsilon^{1-q}\|h\|_{s}
$$

Putting this estimate together with (6.1) and (6.3), we have

$$
\left|\int_{W} \mathcal{L}_{T_{1}} h \varphi d m-\int_{W} \mathcal{L}_{T_{2}} h \varphi d m\right| \leq C\left(\|h\|_{s} \varepsilon^{\alpha}+\|h\|_{u} \varepsilon^{\beta}+\|h\|_{s} \varepsilon^{1-q}\right) \leq C b^{-1} \varepsilon^{\beta}\|h\| .
$$

Taking the supremum over all $W \in \Sigma$ and $\varphi \in \mathcal{C}^{1}(W)$ yields the lemma.

Lemma 6.1 implies $\left\|\left|\mathcal{L}_{T_{1}}-\mathcal{L}_{T_{2}}\right|\right\| \leq C \varepsilon^{\beta}$ whenever $\gamma\left(T_{1}, T_{2}\right) \leq \varepsilon$. Since both $T_{1}$ and $T_{2}$ satisfy the Lasota-Yorke inequalities (2.7)-(2.9), we may apply the results of $\left[\mathrm{KL}\right.$ to our operator $\mathcal{L}: \mathcal{B} \rightarrow \mathcal{B}_{w}$.

6.2. Smooth random perturbations. Recall the transfer operator $\mathcal{L}_{\nu, g}$ associated with the random process defined in Section 2.3. For the remainder of this section, we fix constants $\lambda, \mu, \mu_{+}$and $D_{n}$ such that (2.1) and (2.6) are satisfied for all $\tilde{T} \in X_{\varepsilon}$.

The following is a generalization of Lemma 6.1 which shows that the transfer operator associated with the random perturbation is also close to $\mathcal{L}_{T}$ in the sense of $[\mathrm{KL}]$.

Lemma 6.2. $\left\|\left|\mathcal{L}_{\nu, g}-\mathcal{L}_{T}\right|\right\| \leq C_{b} A \varepsilon^{\beta}$.

Proof. Let $h, \varphi \in \mathcal{C}^{1}(\mathcal{M}),|\varphi|_{\mathcal{C}^{1}} \leq 1$, and $W \in \Sigma$. Then using (6.4) of Lemma 6.1

$$
\begin{aligned}
& \left|\int_{W} \mathcal{L}_{\nu, g} h \varphi d m-\int_{W} \mathcal{L}_{T} h \varphi d m\right| \\
& \quad=\left|\int_{\Omega} \int_{W}\left(\mathcal{L}_{T_{\omega}} h(x)-\mathcal{L}_{T} h(x)\right) \varphi(x) g\left(\omega, T_{\omega}^{-1} x\right) d m d \nu\right| \\
& \quad \leq \int_{\Omega} C_{b} \varepsilon^{\beta}\|h\||g(\omega, \cdot)|_{C^{1}} d \nu(\omega) \leq C_{b} A \varepsilon^{\beta}\|h\| .
\end{aligned}
$$

We next prove uniform Lasota-Yorke estimates for the operator $\mathcal{L}_{\nu, g}$. First, we need to introduce some notation. Let $\bar{\omega}_{n}=\left(\omega_{1}, \ldots, \omega_{n}\right) \in \Omega^{n}$. We define $T_{\bar{\omega}_{n}}=T_{\omega_{n}} \circ \cdots \circ T_{\omega_{1}}$ and similarly $D T_{\bar{\omega}_{n}}=\Pi_{j=1}^{n} D T_{\omega_{j}}\left(T_{\bar{\omega}_{j-1}}\right)$.

Lemma 6.3. Let $\Delta(\nu, g) \leq \varepsilon$. For $\varepsilon$ sufficiently small, there exists $\delta_{0}>0$ and a constant $C=C_{a, A}$, such that for all $h \in \mathcal{B}, \delta \leq \delta_{0}$ and $n \geq 0, \mathcal{L}_{\nu, g}$ satisfies

$$
\begin{aligned}
\left|\mathcal{L}_{\nu, g}^{n} h\right|_{w} & \leq C D^{n}|h|_{w}, \\
\left\|\mathcal{L}_{\nu, g}^{n} h\right\|_{s} & \leq C \max \left\{\rho, \mu_{+}^{q}\right\}^{n} D_{n}\|h\|_{s}+C_{\delta} D_{n}|h|_{w}, \\
\left\|\mathcal{L}_{\nu, g}^{n} h\right\|_{u} & \leq C \lambda^{-\beta n} D_{n}\|h\|_{u}+C\left(D_{n}+L_{n} \lambda^{-n} \mu^{-\alpha n}\right)\|h\|_{s} .
\end{aligned}
$$


Proof. The proofs follow from those of Section 4 except that we have the added function $g(\omega, x)$. Notice that

$$
\mathcal{L}_{\nu, g}^{n} h(x)=\int_{\Omega^{n}} h \circ T_{\bar{\omega}_{n}}^{-1}\left|D T_{\bar{\omega}_{n}}\left(T_{\bar{\omega}_{n}}^{-1}\right)\right|^{-1} \Pi_{j=1}^{n} g\left(\omega_{j}, T_{\omega_{j}}^{-1} \circ \cdots \circ T_{\omega_{n}}^{-1} x\right) d \nu^{n}\left(\bar{\omega}_{n}\right) .
$$

Estimating the strong stable norm. For any $W \in \Sigma$, we define the connected pieces $W_{i}$ of $T_{\bar{\omega}_{n}}^{-1} W$ inductively just as we did for $T^{-n} W$ in Section 3.2. Following the estimates of Section 4.2, we write

$$
\begin{aligned}
& \int_{W} \mathcal{L}_{\nu, g}^{n} h \varphi d m=\int_{\Omega^{n}} \sum_{i}\left\{\int_{W_{i}} h \bar{\varphi}_{i}\left|D T_{\bar{\omega}_{n}}\right|^{-1} J_{W} T_{\bar{\omega}_{n}} \Pi_{j=1}^{n} g\left(\omega_{j}, T_{\bar{\omega}_{j-1}} x\right) d m(x)\right. \\
& \left.\quad+\frac{1}{\left|W_{i}\right|} \int_{W_{i}} \varphi \circ T_{\bar{\omega}_{n}} \int_{W_{i}} h\left|D T_{\bar{\omega}_{n}}\right|^{-1} J_{W} T_{\bar{\omega}_{n}} \Pi_{j=1}^{n} g\left(\omega_{j}, T_{\bar{\omega}_{j-1}} x\right) d m(x)\right\} d \nu^{n}\left(\bar{\omega}_{n}\right)
\end{aligned}
$$

where $\bar{\varphi}_{i}=\varphi \circ T_{\bar{\omega}_{n}}-\frac{1}{\left|W_{i}\right|} \int_{W_{i}} \varphi \circ T_{\bar{\omega}_{n}}$. We fix $\bar{\omega}_{n}$ and define

$$
G_{\bar{\omega}_{n}}(x)=\Pi_{j=1}^{n} g\left(\omega_{j}, T_{\bar{\omega}_{j-1}} x\right) .
$$

To estimate the first sum in (6.5), we note that (4.6) implies

$$
\left|\bar{\varphi}_{i}\right|_{C^{q}\left(W_{i}\right)} \leq C\left|J_{W} T_{\bar{\omega}_{n}}\right|_{C^{0}\left(W_{i}\right)}^{q}|W|^{-\alpha} .
$$

Then, using (4.7), we estimate

$$
\begin{aligned}
& \sum_{i} \int_{W_{i}} h \bar{\varphi}_{i}\left|D T_{\bar{\omega}_{n}}\right|^{-1} J_{W} T_{\bar{\omega}_{n}} G_{\bar{\omega}_{n}} d m \\
& \quad \leq\left.\left.\sum_{i} C\|h\|_{s}\left|W_{i}\right|^{\alpha}|| D T_{\bar{\omega}_{n}}\right|^{-1} J_{W} T_{\bar{\omega}_{n}}\right|_{C^{q}\left(W_{i}\right)}\left|\bar{\varphi}_{i}\right|_{C^{q}\left(W_{i}\right)}\left|G_{\bar{\omega}_{n}}\right|_{C^{q}\left(W_{i}\right)} \\
& \quad \leq\left.\left.\sum_{i} C\|h\|_{s}\left|W_{i}\right|^{\alpha}|| D T_{\bar{\omega}_{n}}\right|^{-1} J_{W} T_{\bar{\omega}_{n}}\right|_{C^{0}\left(W_{i}\right)}\left|J_{W} T_{\bar{\omega}_{n}}\right|_{C^{0}\left(W_{i}\right)}^{q}|W|^{-\alpha}\left|G_{\bar{\omega}_{n}}\right|_{\mathcal{C}^{q}\left(W_{i}\right)} .
\end{aligned}
$$

The only additional term here is $\left|G_{\bar{\omega}_{n}}\right|_{\mathcal{C}^{q}\left(W_{i}\right)}$, which we now show is bounded independently of $n$ and $W_{i}$.

Sublemma 6.4. Let $W_{i} \in \Sigma$ be a smooth component of $T_{\bar{\omega}_{n}}^{-1} W$. There exists a constant $C>0$, independent of $W, n$ and $\bar{\omega}_{n}$ such that

$$
\left|\Pi_{j=1}^{n} g\left(\omega_{j}, T_{\bar{\omega}_{j-1}} \cdot\right)\right|_{\mathcal{C}^{1}\left(W_{i}\right)} \leq C \Pi_{j=1}^{n} g\left(\omega_{j}, T_{\bar{\omega}_{j-1}} x\right)
$$

for any $x \in W_{i}$.

Proof. The proof follows the usual distortion estimates along stable leaves. For any $x, y \in W_{i}$,

$$
\begin{aligned}
\log \frac{\Pi_{j=1}^{n} g\left(\omega_{j}, T_{\bar{\omega}_{j-1}} x\right)}{\prod_{j=1}^{n} g\left(\omega_{j}, T_{\bar{\omega}_{j-1}} y\right)} & \leq \sum_{j=1}^{n} a^{-1}\left|g\left(\omega_{j}, \cdot\right)\right|_{C^{1}\left(W_{i}\right)} d\left(T_{\bar{\omega}_{j-1}} x, T_{\bar{\omega}_{j-1}} y\right) \\
& \leq \sum_{j=1}^{\infty} A a^{-1} C \mu_{+}^{j-1} d(x, y)=: \quad c_{0} d(x, y),
\end{aligned}
$$

using property (iii) of $g$. The distortion bound yields the lemma with $C=c_{0} e^{c_{0}}$. 
The sublemma allows us to estimate (6.6) using (4.7):

$$
\sum_{i} \int_{W_{i}} h \bar{\varphi}_{i}\left|D T_{\bar{\omega}_{n}}\right|^{-1} J_{W} T_{\bar{\omega}_{n}} G_{\bar{\omega}_{n}} d m \leq C\|h\|_{s} D_{n} \mu_{+}^{q n} \Pi_{j=1}^{n} g\left(\omega_{j}, T_{\bar{\omega}_{j-1}} x_{*}\right)
$$

where $x_{*}$ is some point in $T_{\bar{\omega}_{n}}^{-1} W$.

We estimate the second term of (6.5) in a similar way according to (4.8). Each time, we replace $\left|G_{\bar{\omega}_{n}}\right|_{\mathcal{C}^{q}}$ or $\left|G_{\bar{\omega}_{n}}\right|_{\mathcal{C}^{1}}$ according to Sublemma 6.4

$$
\begin{aligned}
\sum_{i} \frac{1}{\left|W_{i}\right|} \int_{W_{i}} \varphi \circ T_{\bar{\omega}_{n}} d m & \int_{W_{i}} h\left|D T_{\bar{\omega}_{n}}\right|^{-1} J_{W} T_{\bar{\omega}_{n}} G_{\bar{\omega}_{n}} d m \\
& \leq\left(C\|h\|_{s} \rho^{n}+C_{\delta} D_{n}|h|_{w}\right) \Pi_{j=1}^{n} g\left(\omega_{j}, T_{\bar{\omega}_{j-1}} x_{*}\right) .
\end{aligned}
$$

Combining this estimate with (6.7), we have

$$
\int_{W} \mathcal{L}_{T_{\varpi_{n}}} h \varphi d m \leq\left(C\|h\|_{s}\left(D_{n} \mu_{+}^{q n}+\rho^{n}\right)+C_{\delta} D_{n}|h|_{w}\right) \Pi_{j=1}^{n} g\left(\omega_{j}, T_{\bar{\omega}_{j-1}} x_{*}\right) .
$$

Now integrating this expression over $\Omega^{n}$, we integrate one $\omega_{j}$ at a time starting with $\omega_{n}$. Note that $\int_{\Omega} g\left(\omega_{n}, T_{\bar{\omega}_{n-1}} x_{*}\right) d \nu\left(\omega_{n}\right)=1$ by assumption on $g$ since $T_{\bar{\omega}_{n-1}} x_{*}$ is independent of $\omega_{n}$. Similarly, each factor in $G_{\bar{\omega}_{n}}$ integrates to 1 so that

$$
\left\|\mathcal{L}_{\nu, g}^{n} h\right\|_{s} \leq C\|h\|_{s}\left(D_{n} \mu_{+}^{q n}+\rho^{n}\right)+C_{\delta} D_{n}|h|_{w}
$$

which is the Lasota-Yorke inequality for the strong stable norm.

The inequalities for the strong unstable norm and for the weak norm follow almost identically, always using Sublemma 6.4.

6.3. Hyperbolic systems with holes. We adopt the notation and conditions introduced in Section 2.5. The first lemma shows that we can make the operators $\mathcal{L}$ and $\mathcal{L}_{H}$ arbitrarily close by controlling the "diameter" $r$ of the hole along elements of $\Sigma$ and the number $P$ of connected components of the hole that a leaf can intersect at time 1 .

Lemma 6.5. Let $H$ be a hole satisfying assumption (H1). There exists $C>0$ depending only on $T$ such that

$$
\left\|\mathcal{L}-\mathcal{L}_{H} \mid\right\| \leq C \operatorname{Pr}^{\alpha}
$$

Proof. Let $h \in \mathcal{C}^{1}(\mathcal{M}), W \in \Sigma$ and $\varphi \in \mathcal{C}^{1}(W)$ with $|\varphi|_{\mathcal{C}^{1}(W)} \leq 1$. Recall that $\mathcal{M}^{1} \subset \mathcal{M} \backslash H$ is the set of points which remains in $\mathcal{M}$ until at least time 1 . Let $1_{\mathcal{M} \backslash \mathcal{M}^{1}}$ denote the indicator function of $\mathcal{M} \backslash \mathcal{M}^{1}$ :

$$
\begin{aligned}
\int_{W}(\mathcal{L} & \left.-\mathcal{L}_{H}\right) h \varphi d m=\int_{W} \mathcal{L}\left(1_{\mathcal{M} \backslash \mathcal{M}^{1}} h\right) \varphi d m \\
& =\int_{T^{-1} W \cap \mathcal{M} \backslash \mathcal{M}^{1}} h \varphi \circ T|D T|^{-1} J_{W} T d m \\
& \leq\left.\left.\sum_{\tilde{W}_{i}}\|h\|_{s}\left|\tilde{W}_{i}\right|^{\alpha}|\varphi \circ T|_{\mathcal{C}^{q}\left(\tilde{W}_{i}\right)}|| D T\right|^{-1} J_{W} T\right|_{\mathcal{C}^{0}\left(\tilde{W}_{i}\right)}
\end{aligned}
$$

where $\tilde{W}_{i}$ are the connected components of $T^{-1} W \cap \mathcal{M} \backslash \mathcal{M}^{1}$, i.e. the pieces of $T^{-1} W$ which are in the hole at time 0 or 1 . We recall from the estimates of 
Section 4 that $|\varphi \circ T|_{\mathcal{C}^{q}\left(\tilde{W}_{i}\right)} \leq|\varphi|_{\mathcal{C}^{q}(W)}$. Also, the distortion bound A.1 implies $\left|J_{W} T\right|\left|\tilde{W}_{i}\right| \leq C\left|T \tilde{W}_{i}\right|$. We then have

$$
\int_{W}\left(\mathcal{L}-\mathcal{L}_{H}\right) h \varphi d m \leq C\|h\|_{s} \sum_{i}\left|T \tilde{W}_{i}\right|^{\alpha} \leq C\|h\|_{s} P r^{\alpha},
$$

which completes the proof of the lemma.

The next proposition proves uniform Lasota-Yorke estimates for $\mathcal{L}_{H}$ which are independent of $H$ satisfying assumptions (H1) and (H2). Once it is proven, we may use it in combination with Lemma 6.5 to invoke the results of $\mathrm{KL}$ and conclude that the spectra of $\mathcal{L}$ and $\mathcal{L}_{H}$ are close if $r$ is small. This proves Theorem 2.18,

Proposition 6.6. Let $H$ be a hole satisfying assumptions (H1) and (H2) of Section 2.5 and let $\rho_{1}:=\frac{L+P}{\lambda \mu^{\alpha}}<1$. Choose $\beta \leq \alpha / 2$. There exists $\delta_{0}>0$, depending only on $P$, such that for all $h \in \mathcal{B}, \delta \leq \delta_{0}$ and $n \geq 0, \mathcal{L}_{H}$ satisfies

$$
\begin{aligned}
\left|\mathcal{L}_{H}^{n} h\right|_{w} & \leq C D_{n}|h|_{w} \\
\left\|\mathcal{L}_{H}^{n} h\right\|_{s} & \leq C \max \left\{\rho_{1}, \mu_{+}^{q}\right\}^{n} D_{n}\|h\|_{s}+C_{\delta} D_{n}|h|_{w} \\
\left\|\mathcal{L}_{H}^{n} h\right\|_{u} & \leq C \lambda^{-\beta n} D_{n}\|h\|_{u}+C\left(D_{n}+\left(L_{n}+P_{n}\right) \lambda^{-n} \mu^{-\alpha n}\right)\|h\|_{s} .
\end{aligned}
$$

Proof. Our estimates follow closely those of Section 4, so to avoid repetition we indicate only where the presence of the holes requires us to modify those estimates. First notice that Lemmas 3.1 and 3.2 hold for the map with holes with $\rho_{1}$ in place of $\rho$. This is because the definition of the elements $W_{i}^{k}$ of $\mathcal{W}_{k}$ and their tree-like structure remains unchanged. The number of connected components of $\tilde{T}^{-n} W$ may be greater, but the growth of the number of short pieces is controlled by assumption (H2). Summing up to most recent long ancestors as we did in the proof of Lemma 3.1 and using (H2), we see that equation (3.1) becomes

$$
\left.\left.\sum_{i \in J_{n}\left(W_{j}^{k}\right)}\left|W_{i}^{n}\right|^{\varsigma}|| D T^{n}\right|^{-1} J_{W} T^{n}\right|_{\mathcal{C}^{0}\left(W_{i}^{n}\right)} \leq\left.\left. C|| D T^{k}\right|^{-1} J_{W} T^{k}\right|_{\mathcal{C}^{0}\left(W_{j}^{k}\right)}\left|W_{j}^{k}\right|^{\varsigma} \rho_{1}^{n-k} .
$$

The proof of expressions analogous to equations (3.2)-(3.4) is now identical to the proof of Lemma 3.1. We conclude that

$$
\left.\left.\sum_{i}\left|W_{i}^{n}\right|^{\varsigma}|| D T^{n}\right|^{-1} J_{W} T^{n}\right|_{\mathcal{C}^{0}\left(W_{i}^{n}\right)} \leq C D_{n} \delta^{\varsigma-\alpha}|W|^{\alpha}+C|W|^{\varsigma} \rho_{1}^{n} .
$$

Estimating the weak norm. For any $h \in \mathcal{C}^{1}(\mathcal{M}), W \in \Sigma$ and $\varphi \in \mathcal{C}^{1}(W)$ with $|\varphi|_{C^{1}(W)} \leq 1$, we have

$$
\begin{aligned}
\int_{W} \mathcal{L}_{H}^{n} h \varphi d m & =\sum_{W_{i} \in \mathcal{W}_{n}} \int_{W_{i}} h\left|D T^{n}\right|^{-1} J_{W} T^{n} \varphi \circ T^{n} d m \\
& \leq\left.\left. C|h|_{w} \sum_{W_{i} \in \mathcal{W}_{n}}|| D T^{n}\right|^{-1} J_{W} T^{n}\right|_{\mathcal{C}^{0}\left(W_{i}^{n}\right)} \leq C D_{n}|h|_{w}
\end{aligned}
$$

where in the last inequality we have used (6.11) with $\varsigma=0$. This proves (6.8).

Estimating the strong stable norm. As in Section 4.2, we define $\bar{\varphi}_{i}=\varphi \circ T^{n}-$ $\frac{1}{\left|W_{i}\right|} \int_{W_{i}} \varphi \circ T^{n}$. Equation (4.7) remains unchanged,

$$
\sum_{i} \int_{W_{i}} h\left|D T^{n}\right|^{-1} J_{W} T^{n} \bar{\varphi}_{i} d m \leq C\|h\|_{s} D_{n} \mu_{+}^{q n} .
$$


The estimate for equation (4.8) is modified slightly according to (6.11),

$$
\sum_{i} \frac{1}{\left|W_{i}\right|} \int_{W_{i}} \varphi \circ T^{n} d m \int_{W_{i}} h\left|D T^{n}\right|^{-1} J_{W} T^{n} d m \leq C\|h\|_{s} \rho_{1}^{n}+C_{\delta} D_{n}|h|_{w} .
$$

Combining these two estimates, we see that

$$
\left\|\mathcal{L}_{H}^{n} h\right\|_{s} \leq C\|h\|_{s}\left(D_{n} \mu_{+}^{q n}+\rho_{1}^{n}\right)+C_{\delta} D_{n}|h|_{w},
$$

which proves (6.9).

Estimating the strong unstable norm. Given two admissible leaves $W^{1}$ and $W^{2}$ satisfying $d_{\Sigma}\left(W^{1}, W^{2}\right) \leq \varepsilon$, we partition them into long pieces $U_{j}^{i}$ and short pieces $V_{k}^{i}$ as in Section 4.3 where for each $j$, the pieces $U_{j}^{1}$ and $U_{j}^{2}$ are paired up so that $d_{\Sigma}\left(T^{-n} U_{j}^{1}, T^{-n} U_{j}^{2}\right) \leq C \lambda^{-n} \varepsilon$. The introduction of the hole only increases the number of unpaired pieces $V_{k}^{i}$ : if part of $T^{-n} W^{1}$ has fallen in the hole while the corresponding part of $T^{-n} W^{2}$ has not, then a piece $V_{k}^{2} \subset W^{2}$ is created. We estimate the size of $V_{k}^{2}$ using assumption (H1).

Suppose the part of $W^{1}$ corresponding to $V_{k}^{2}$ falls in the hole at time $\ell \leq n$. If the boundary of the hole at that point is strictly convex with curvature greater than $B$, then $\left|T^{-\ell} V_{k}^{2}\right| \leq C \sqrt{\varepsilon}$ and so $\left|V_{k}^{2}\right| \leq C \sqrt{\varepsilon}$ as well. On the other hand, if the boundary of the hole is transverse to the stable cone, then the estimate improves to $\left|T^{-\ell} V_{k}^{2}\right| \leq C \varepsilon$. Notice also that there can be at most $L_{n}+P_{n}+2$ pieces $V_{k}^{i}$.

Using this bound on the $V_{k}^{i}$, (4.10) becomes

$$
\begin{aligned}
\sum_{i, k} \int_{T^{-n} V_{k}^{i}} h\left|D T^{n}\right|^{-1} J_{W} T^{n} \varphi_{i} \circ T^{n} d m & \leq\left.\left. C\|h\|_{s} \sum_{i, k}\left|V_{k}^{i}\right|^{\alpha}|| D T^{n}\right|^{-1}\left(J_{W} T^{n}\right)^{1-\alpha}\right|_{C^{0}} \\
& \leq C \varepsilon^{\alpha / 2}\|h\|_{s}\left(L_{n}+P_{n}\right) \lambda^{-n} \mu^{-\alpha n} .
\end{aligned}
$$

The estimates on the paired pieces $U_{j}^{i}$ do not change, so putting together equation (6.12) with (4.14) and (4.22) and using the fact that $\alpha / 2 \geq \beta$, we have

$$
\left\|\mathcal{L}_{H}^{n} h\right\|_{u} \leq C \lambda^{-\beta n} D_{n}\|h\|_{u}+C\|h\|_{s}\left(D_{n}+\left(L_{n}+P_{n}\right) \lambda^{-n} \mu^{-\alpha n}\right) .
$$

This completes the proof of (6.10).

\section{Appendix A. Distortion bounds}

The following are distortion bounds used in deriving the Lasota-Yorke estimates which are standard for uniformly hyperbolic $\mathcal{C}^{2}$ maps. For any $n \in \mathbb{N}$ and $x, y \in$ $K \in \mathcal{K}_{n}$ the following estimates hold:

$$
\begin{aligned}
& \left|\frac{\left|D T^{n}(x)\right|}{\left|D T^{n}(y)\right|}-1\right| \leq C \max \left\{d(x, y), d\left(T^{n} x, T^{n}, y\right)\right\}, \\
& \left|\frac{J_{W} T^{n}(x)}{J_{W} T^{n}(y)}-1\right| \leq C \max \left\{d(x, y), d\left(T^{n} x, T^{n}, y\right)\right\} .
\end{aligned}
$$

In particular, these bounds imply that $\left.\left.|| D T^{n}\right|^{-1}\right|_{C^{q}\left(W_{i}\right)} \leq\left.\left. C|| D T^{n}\right|^{-1}\right|_{\mathcal{C}^{0}\left(W_{i}\right)}$ and similarly $\left|J_{W} T^{n}\right|_{C^{q}\left(W_{i}\right)} \leq C\left|J_{W} T^{n}\right|_{\mathcal{C}^{0}\left(W_{i}\right)}$ for any $0 \leq q \leq 1$.

Note that for $x \in T^{-n} W,\left|D T^{n}(x)\right|=C_{\theta}(x) J_{W} T^{n}(x) J_{u} T^{n}(x)$ where $J_{u} T^{n}$ is the Jacobian of $T^{n}$ in the unstable direction and $C_{\theta}(x)$ is a number which depends on the angle between the unstable direction and $T^{-n} W$ at the point $x$. Since the family of admissible leaves $W$ is uniformly transversal to the unstable direction, 
there exists a constant $c_{0}>0$, independent of $W$, such that $\left|C_{\theta}\right| \geq c_{0}$. Thus for all $n \geq 0$

$$
\left.\left.|| D T^{n}\right|^{-1} J_{W} T^{n}\right|_{\infty} \leq C \lambda^{-n}
$$

wherever $\left|D T^{n}\right|$ is defined.

\section{REFERENCES}

[Ba] V.I. Bakhtin, A direct method for constructing an invariant measure on a hyperbolic attractor. (Russian) Izv. Ross. Akad. Nauk Ser. Mat. 56 (1992), 934-957; English transl., Russian Acad. Sci. Izv. Math. 41:2 (1993), 207-227. MR1209028 (94d:58083)

[B1] V. Baladi, Positive transfer operators and decay of correlations, Advanced Series in Nonlinear Dynamics, 16, World Scientific (2000). MR.1793194 (2001k:37035)

[B2] V. Baladi, Anisotropic Sobolev spaces and dynamical transfer operators: $\mathcal{C}^{\infty}$ foliations, Algebraic and Topological Dynamics, Sergiy Kolyada, Yuri Manin and Tom Ward, eds. Contemporary Mathematics, Amer. Math. Society, (2005) 123-136. MR2180233 (2007c:37022)

[BT] V. Baladi, M. Tsujii, Anisotropic Hölder and Sobolev spaces for hyperbolic diffeomorphisms, Ann. Inst. Fourier 57 (2007), 127-154. MR2313087 (2008d:37034)

[BY] V. Baladi, L.-S. Young, On the spectra of randomly perturbed expanding maps, Comm. Math. Phys. 156:2 (1993), 355-385; 166:1 (1994), 219-220. MR1233850 (94g:58172)

[BC] H. van den Bedem and N. Chernov, Expanding maps of an interval with holes, Ergod. Th. and Dynam. Sys. 22 (2002), 637-654. MR1908547 (2003c:37046)

[BKL] M. Blank, G. Keller, C. Liverani, Ruelle-Perron-Frobenius spectrum for Anosov maps, Nonlinearity 15:6 (2001), 1905-1973. MR1938476 (2003m:37033)

$[\mathrm{Bu}] \quad$ J. Buzzi, Absolutely continuous invariant probability measures for arbitrary expanding piecewise $\mathbb{R}$-analytic mappings of the plane, Ergod. Th. and Dynam. Sys. 20:3 (2000), 697-708. MR1764923 (2001h:37070)

[BK] J. Buzzi and G. Keller, Zeta functions and transfer operators for multidimensional piecewise affine and expanding maps, Ergod. Th. and Dynam. Sys. 21:3 (2001), 689-716. MR1836427 (2002d:37036)

[C] N. N. Cencova, A natural invariant measure on Smale's horseshoe, Soviet Math. Dokl. 23 (1981), 87-91.

[CG] J.-R. Chazottes and S. Gouezel, On almost-sure versions of classical theorems for dynamical systems, Probability Theory and Related Fields 138 (2007), 195-234. MR2288069 (2008a:60066)

[Ch] N. Chernov, Advanced statistical properties of dispersing billiards, J. Stat. Phys. 122 (2006), 1061-1094. MR2219528 (2007h:37047)

[CD] N. Chernov, D.Dolgopyat, Brownian Brownian Motion - I, to appear in Memoirs of AMS.

[CM1] N. Chernov and R. Markarian, Ergodic properties of Anosov maps with rectangular holes, Bol. Soc. Bras. Mat. 28 (1997), 271-314. MR1479505 (98k:58140)

[CM2] N. Chernov and R. Markarian, Anosov maps with rectangular holes. Nonergodic cases, Bol. Soc. Bras. Mat. 28 (1997), 315-342. MR.1479506 (99b:58142)

[CMT1] N. Chernov, R. Markarian and S. Troubetskoy, Conditionally invariant measures for Anosov maps with small holes, Ergod. Th. and Dynam. Sys. 18 (1998), 1049-1073. MR.1653291 (99k:58111)

[CMT2] N. Chernov, R. Markarian and S. Troubetskoy, Invariant measures for Anosov maps with small holes, Ergod. Th. and Dynam. Sys. 20 (2000), 1007-1044. MR.1779391 (2001f:37042)

[CY] N. Chernov and L.-S. Young, Decay of correlations for Lorentz gases and hard balls, in Hard Ball Systems and the Lorentz Gas, D. Szasz, ed., Enclyclopaedia of Mathematical Sciences 101, Springer-Verlag, Berlin, 2000, 89-120. MR.1805327 (2001k:37046)

[D1] M. Demers, Markov Extensions for Dynamical Systems with Holes: An Application to Expanding Maps of the Interval, Israel J. of Math. 146 (2005), 189-221. MR2151600 (2006d:37062) 
[D2] M. Demers, Markov Extensions and Conditionally Invariant Measures for Certain Logistic Maps with Small Holes, Ergod. Th. and Dynam. Sys. 25:4 (2005), 1139-1171. MR:2158400 (2007d:37057)

[DY] M. Demers and L.-S. Young, Escape rates and natural conditionally invariant measures, Nonlinearity 19 (2006), 377-397. MR.2199394(2006i:37051)

[GL] S. Gouëzel and C. Liverani, Banach spaces adapted to Anosov systems, Ergod. Th. and Dynam. Sys., 26, 1, 189-217 (2006). MR2201945 (2007h:37037)

[HH] H. Hennion and L.Hevré, Limit theorems for Markov chains and stochastic properties of dynamical systems by quasi-compactness, 1766, Lectures Notes in Mathematics, Springer-Verlag, Berlin, 2001. MR:1862393(2002h:60146)

[K] G. Keller On the rate of convergence to equilibrium in one-dimensional systems, Comm. Math. Phys. 96 (1984), no. 2, 181-193. MR768254 (86k:58071)

[KL] G. Keller and C. Liverani, Stability of the spectrum for transfer operators, Annali della Scuola Normale Superiore di Pisa, Scienze Fisiche e Matematiche (4) XXVIII (1999), 141-152. MR.1679080 (2000b:47030)

[LY] A. Lasota and J.A. Yorke, On the existence of invariant measures for piecewise monotonic transformations, Trans. Amer. Math. Soc. 186 (1963), 481-488. MR0335758 (49:538)

[L1] C. Liverani, Decay of Correlations, Annals of Mathematics 142 (1995), 239-301. MR.1343323 (96e:58090)

[L2] C. Liverani, Invariant measures and their properties. A functional analytic point of view, Dynamical Systems. Part II: Topological Geometrical and Ergodic Properties of Dynamics. Pubblicazioni della Classe di Scienze, Scuola Normale Superiore, Pisa. Centro di Ricerca Matematica "Ennio De Giorgi": Proceedings. Published by the Scuola Normale Superiore in Pisa (2004). MR2071241 (2005d:37045)

[L3] C. Liverani, Fredholm determinants, Anosov maps and Ruelle resonances, Discrete and Continuous Dynamical Systems 13:5 (2005), 1203-1215. MR2166265(2006k:37047)

[LiM] C. Liverani and V. Maume-Deschamps, Lasota-Yorke maps with holes: conditionally invariant probability measures and invariant probability measures on the survivor set, Annales de l'Institut Henri Poincaré Probability and Statistics 39 (2003), 385-412. MR.1978986 (2004c:37009)

[LW] C. Liverani and M. Wojtkowski, Ergodicity in Hamiltonian Systems, Dynamics Reported 4 (1995), 130-202. MR1346498 (96g:58144)

[LM] A. Lopes and R. Markarian, Open billiards: cantor sets, invariant and conditionally invariant probabilities, SIAM J. Appl. Math. 56 (1996), 651-680. MR1381665|(97a:58108)

[PP1] W. Parry and M. Pollicott, An analogue of the prime number theorem for closed orbits of Axiom A flows, Annals of Math. 118:3 (1983), 573-591. MR727704 (85i:58105)

[PP2] W. Parry and M. Pollicott, Zeta functions and the periodic orbit structure of hyperbolic dynamics, Astérisque No. 187-188 (1990), 268 pp. MR1085356 (92f:58141)

[P] Ya.B.Pesin, Dynamical systems with generalized hyperbolic attractors: hyperbolic, ergodic and topological properties, Ergod. Th. and Dynam. Sys. 12 (1992), 123-151. MR1162404 (93b:58095)

[Ru1] D. Ruelle, Locating resonances for Axiom A dynamical systems, J. Stat. Phys. 44:3-4 (1986), 281-292. MR857060 (87k:58214)

[Ru2] D. Ruelle, Resonances for Axiom A flows, J. Differential Geom. 25:1 (1987), 99-116. MR873457 (88j:58098)

[R1] H.H. Rugh, The correlation spectrum for hyperbolic analytic maps, Nonlinearity 5:6 (1992), 1237-1263. MR1192517 (93i:58121)

[R2] H.H. Rugh, Fredholm determinants for real-analytic hyperbolic diffeomorphisms of surfaces. XIth International Congress of Mathematical Physics (Paris, 1994), 297-303, Internat. Press, Cambridge, MA, 1995. MR1370685 (96k:58182)

[R3] H.H. Rugh, Generalized Fredholm determinants and Selberg zeta functions for Axiom A dynamical systems. Ergod. Th. and Dynam. Sys. 16:4 (1996), 805-819. MR1406435 (97j:58125)

[S] B. Saussol, Absolutely continuous invariant measures for multidimensional expanding maps, Israel J. Math. 116 (2000), 223-248. MR1759406 (2001e:37037)

[T1] M. Tsujii, Absolutely continuous invariant measures for piecewise real-analytic expanding maps on the plane, Comm. Math. Phys. 208:3 (2000), 605-622. MR.1736328(2000i:37034) 
[T2] M. Tsujii, Absolutely continuous invariant measures for expanding piecewise linear maps, Invent. Math. 143:2 (2001), 349-373. MR1835391 (2002g:37026)

[Y] L.-S. Young, Statistical properties of dynamical systems with some hyperbolicity, Annals of Math. 147:3 (1998), 585-650. MR.1637655 (99h:58140)

Department of Mathematics, Fairfield University, Fairfield, Connecticut 06824

E-mail address: mdemers@mail.fairfield.edu

Dipartimento di Matematica, Università di Roma Tor Vergata, Via della Ricerca SciENTIFICA, 00133 Roma, ITALY

E-mail address: liverani@mat.uniroma2.it 